Tumour-associated antigen A protein derived from tumour cells that can be recognized by the immune system. An immune response may be triggered because a T cell has survived negative thymic selection for the cognate antigen, or more probably because the normal host protein has been mutated within the cancer cell.

\section{Danger signals}

Nuclear and cytosolic proteins that are released by cells during tissue injury or necrosis, and stimulate the innate and adaptive immune system.

Rutgers Cancer Institute of New Jersey, 195 Little Albany Street, Room 2004, New Brunswick, New Jersey 08901, USA Correspondence to H.L.K. e-mail: howard.kaufman@ rutgers.edu

doi: $10.1038 /$ nrd4663 Corrected online 18 January 2016

\title{
Oncolytic viruses: a new class of immunotherapy drugs
}

Howard L. Kaufman, Frederick J. Kohlhapp and Andrew Zloza

Abstract | Oncolytic viruses represent a new class of therapeutic agents that promote anti-tumour responses through a dual mechanism of action that is dependent on selective tumour cell killing and the induction of systemic anti-tumour immunity. The molecular and cellular mechanisms of action are not fully elucidated but are likely to depend on viral replication within transformed cells, induction of primary cell death, interaction with tumour cell antiviral elements and initiation of innate and adaptive anti-tumour immunity. A variety of native and genetically modified viruses have been developed as oncolytic agents, and the approval of the first oncolytic virus by the US Food and Drug Administration (FDA) is anticipated in the near future. This Review provides a comprehensive overview of the basic biology supporting oncolytic viruses as cancer therapeutic agents, describes oncolytic viruses in advanced clinical trials and discusses the unique challenges in the development of oncolytic viruses as a new class of drugs for the treatment of cancer.

Oncolytic virus immunotherapy is a therapeutic approach to cancer treatment that utilizes native or genetically modified viruses that selectively replicate within tumour cells. The ability of viruses to kill cancer cells has been recognized for nearly a century, but only over the past decade have clinical trials documented a therapeutic benefit in patients with cancer ${ }^{1-3}$. Interest in oncolytic viruses has been increasing, based on a better understanding of viral biology, tumour immunology and molecular genetics. Furthermore, a recent randomized Phase III clinical trial demonstrated an improved durable response rate for patients with advanced melanoma who were treated with a modified herpes simplex virus type 1 (HSV-1), encoding granulocyte-macrophage colony-stimulating factor (GM-CSF) ${ }^{3}$. This virus, termed talimogene laherparepvec (T-VEC; Amgen), is widely anticipated to be the first oncolytic virus immunotherapy to be approved by the US Food and Drug Administration (FDA) for the treatment of cancer. The success of T-VEC is likely to promote further drug development within this new class of cancer therapeutics.

Of the nearly 1 million vertebrate viruses, approximately 320,000 are thought to infect mammalian cells ${ }^{4}$. Viruses have several shared properties; these include a genetic element composed of single- or double-stranded DNA or RNA and the ability to infect host cells and replicate under permissive conditions (TABLES 1,2).
The outcome of viral infections can be highly variable depending on the pathogenic nature of the virally encoded genes, interactions between the virus and the host immune system and the ability of the virus to replicate and/or induce latency following infection. Insights into the mechanisms of viral entry, replication, induction and/or suppression of immune responses and lytic versus latent infections have led to an intense interest in utilizing viruses for the treatment of human diseases and have been used to select oncolytic vectors for the treatment of specific types of cancers. In contrast to standard viral-based 'vaccines', oncolytic viruses directly infect and lyse tumour cells in situ. They do not necessarily require defined antigens to be included in the vector as tumour-associated antigens may be released by dying tumour cells. Oncolytic viruses can also provide additional danger signals that can promote an efficient antitumour immune response.

Although incompletely understood, oncolytic viruses are thought to mediate antitumour activity through two distinct mechanisms of action: selective replication within neoplastic cells, resulting in a direct lytic effect on tumour cells; and induction of systemic antitumour immunity. The relative contribution of these mechanisms may differ depending on the nature and type of cancer cell, the characteristics of the viral vector, and the interaction between the virus, tumour microenvironment and host immune system. 
Table 1 | Properties of select DNA viruses

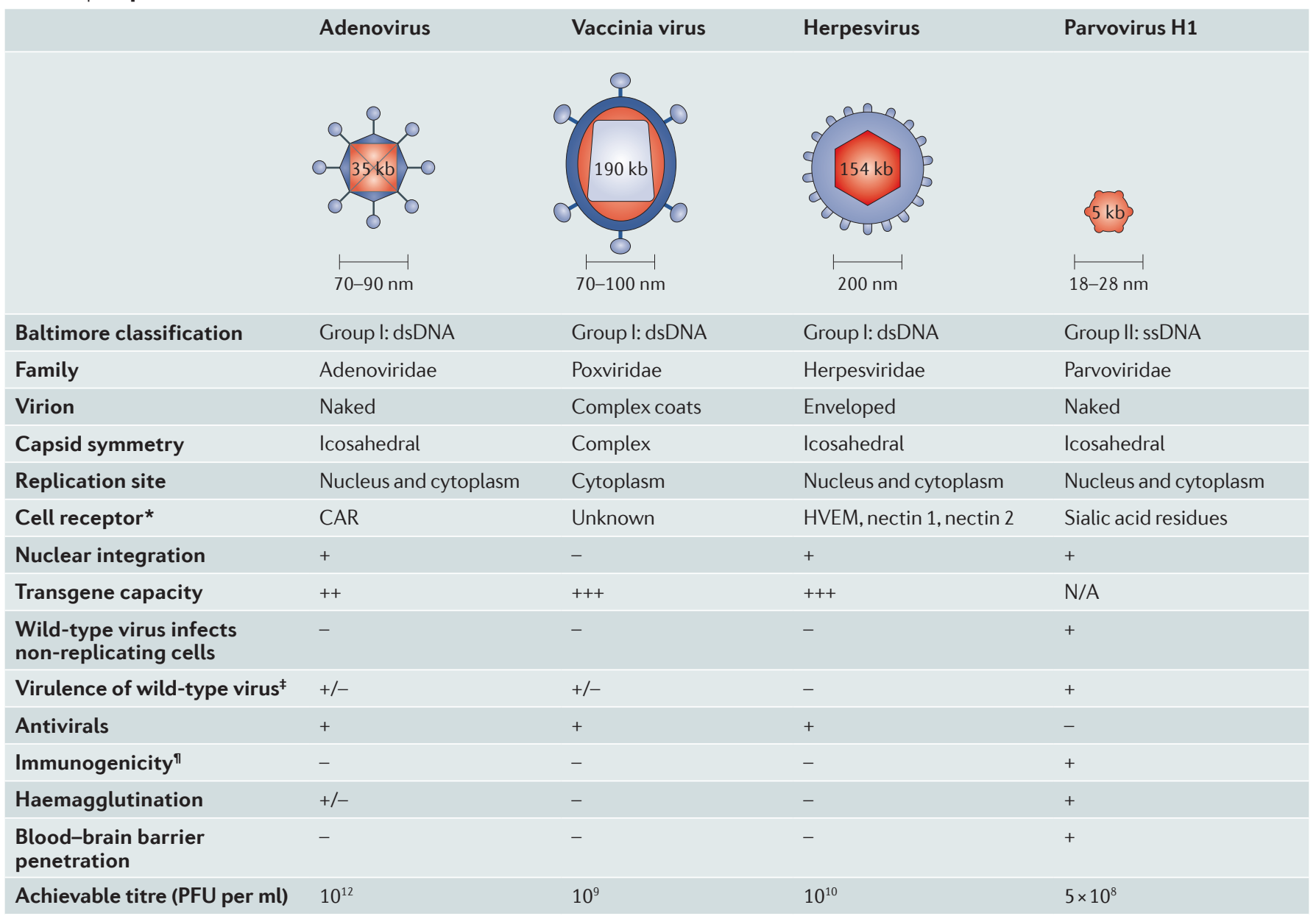

CAR, coxsackie-adenovirus receptor; dsDNA, double-stranded DNA; HVEM, herpesvirus entry mediator; MTD, maximum tolerated dose; N/A, not applicable; PFU, plaque forming unit; ssDNA, single-stranded DNA. *Only well-validated receptors included, others may have been reported. ${ }^{*}$ In humans. "Upon re-exposure.

Certain viruses have the ability to enter cancer cells and selectively replicate within such cells. Although oncolytic viruses can enter both normal and cancer cells, the inherent abnormalities in the cancer cell response to stress, cell signalling and homeostasis provide a selective advantage for viral replication ${ }^{5}$. The normal host cell antiviral machinery, which is responsible for the detection and clearance of viruses, may also be abnormal in cancer cells. For example, the protein kinase $\mathrm{R}(\mathrm{PKR})$ is a critical factor that helps in clearing intracellular viral infections. PKR may be absent in some cancer cells, allowing increased viral replication, whereas it may be active in other cancer cells, such as low-grade tumours, and these differences can influence the therapeutic activity of an oncolytic virus.

The immune response to oncolytic viruses appears to be an important component of the antitumour effect, but it can be a double-edged sword. On the one hand, viruses can help to promote an immune response against the tumour cells by allowing tumour antigen presentation in the context of an active viral infection. On the other hand, neutralizing antiviral responses may block virus replication and ongoing infection of tumour cells. The therapeutic outcome depends on a complex interplay between these opposing forces. When systemic immunity is fully engaged, however, therapeutic responses may be seen in both locally injected tumours and at distant sites of un-infected tumour growth.

Many viruses have been proposed as vectors for oncolytic virus immunotherapy, and considerable work has been done to optimize viral vectors by attenuating pathogenicity and enhancing immunogenicity ${ }^{6}$. To date, adenoviruses, poxviruses, HSV-1, coxsackieviruses, poliovirus, measles virus, Newcastle disease virus (NDV), reovirus, and others, have entered into earlyphase clinical trials. Two viruses, T-VEC and H101 (Shanghai Sunway Biotech) have now achieved regulatory review. $\mathrm{H} 101$ is a genetically modified oncolytic adenovirus that, in combination with chemotherapy, was approved for the treatment of nasopharyngeal carcinoma in China in November 2005 (REFS 3,7).

This Review provides a comprehensive overview of critical issues in the development of oncolytic virus immunotherapy. We discuss preclinical and clinical data that support a role for oncolytic viruses in cancer therapy and detail some of the unique challenges in oncolytic viruse drug development. 
Table 2 | Properties of select RNA viruses

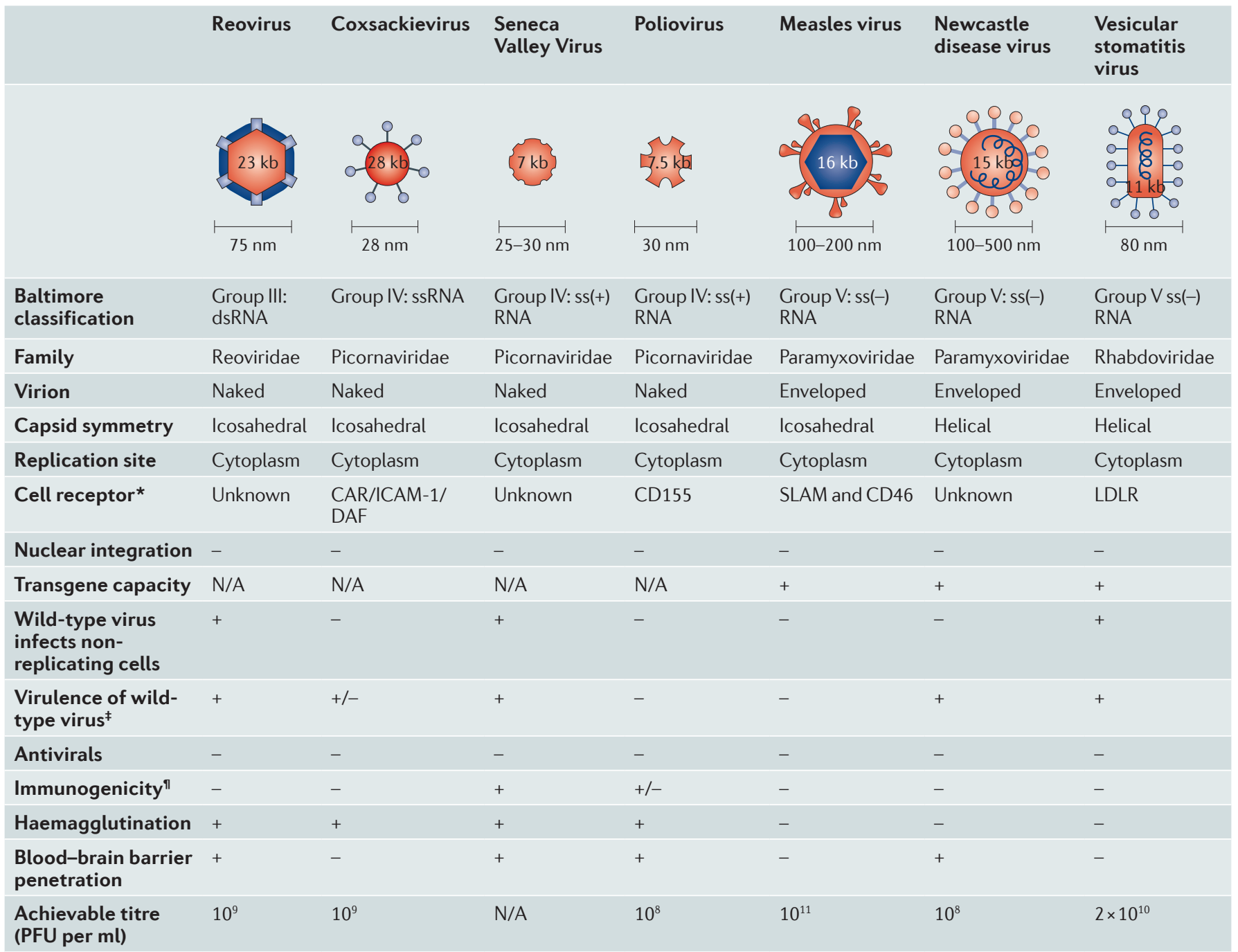

CAR, coxsackie-adenovirus receptor; DAF, decay accelerating factor; dsRNA, double-stranded RNA; ICAM-1, intercellular adhesion molecule 1; LDLR, low-density lipoprotein receptor; MTD, maximum tolerated dose; N/A, not applicable; PFU, plaque forming unit; SLAM, signalling lymphocytic activation molecule; ss(+)RNA, positive single-stranded RNA; ss(-)RNA, negative single-stranded RNA; VP, viral particle. * Only well-validated receptors included, others may have been reported. 和o humans. "Upon re-exposure.

Cell antiviral response elements

Intracellular and extracellular components of the inherent cellular response to viral infection, including detection (via protein kinase R (PKR), Toll-like receptors (TLRs), retinoic acid-inducible gene 1 (RIG-1), and others) and initiation of the immune response through the release of cytokines (such as type I interferons), danger-associated molecular pattern signals (DAMPs) (such as high mobility group box 1 (HMGB1),

heat shock proteins (HSPs), and others), and pathogenassociated molecular patterns (PAMPs; viral products recognized by TLRs).
Mechanisms of oncolytic virus immunotherapy The mechanisms through which oncolytic viruses mediate tumour rejection are incompletely understood. Most oncolytic viruses directly kill host tumour cells. This activity is influenced by the efficiency of cell receptor targeting, viral replication and host cell antiviral response elements ${ }^{8,9}$. The lytic potential of oncolytic viruses also depends on the type of virus, dose, natural and induced viral tropism, and the susceptibility of the cancer cell to the different forms of cell death (apoptosis, necrosis, pyroptosis and autophagy).

In normal cells, a variety of signalling pathways operate to detect and clear pathogenic viral particles (FIG. 1). These pathways can be stimulated by local interferon (IFN) release or through intracellular Toll-like receptors (TLRs), which are activated by viral elements. TLRs are cell surface and intracellular pattern recognition receptors that are activated in response to repeated sequences, so-called pathogen-associated molecular patterns (PAMPs), which are common to pathogenic bacteria and viruses. PAMPs may include elements of viral capsids, DNA, RNA and viral protein products. TLR signalling activates host cell antiviral responses and systemic innate immunity. Several downstream host cell factors involved in oncolytic virus clearance have been identified, including TNFassociated factor 3 (TRAF3), IFN-related factor 3 (IRF3), IRF7 and retinoic acid-inducible gene 1 (RIG-1). These factors activate the JAK-STAT (Janus kinase-signal transducer and activator of transcription) pathway, which coordinates the antiviral machinery in infected cells. The antiviral machinery reinforces local IFN release, which activates PKR activity. PKR is an intracellular protein kinase that recognizes double-stranded RNA and other viral elements ${ }^{10,11}$. When activated by viral elements, PKR terminates cell protein synthesis and promotes rapid cell death and viral clearance. In cancer cells, IFN pathway signalling and PKR activity may be abnormal, and viral clearance is thwarted ${ }^{12}$. 
a Healthy cell

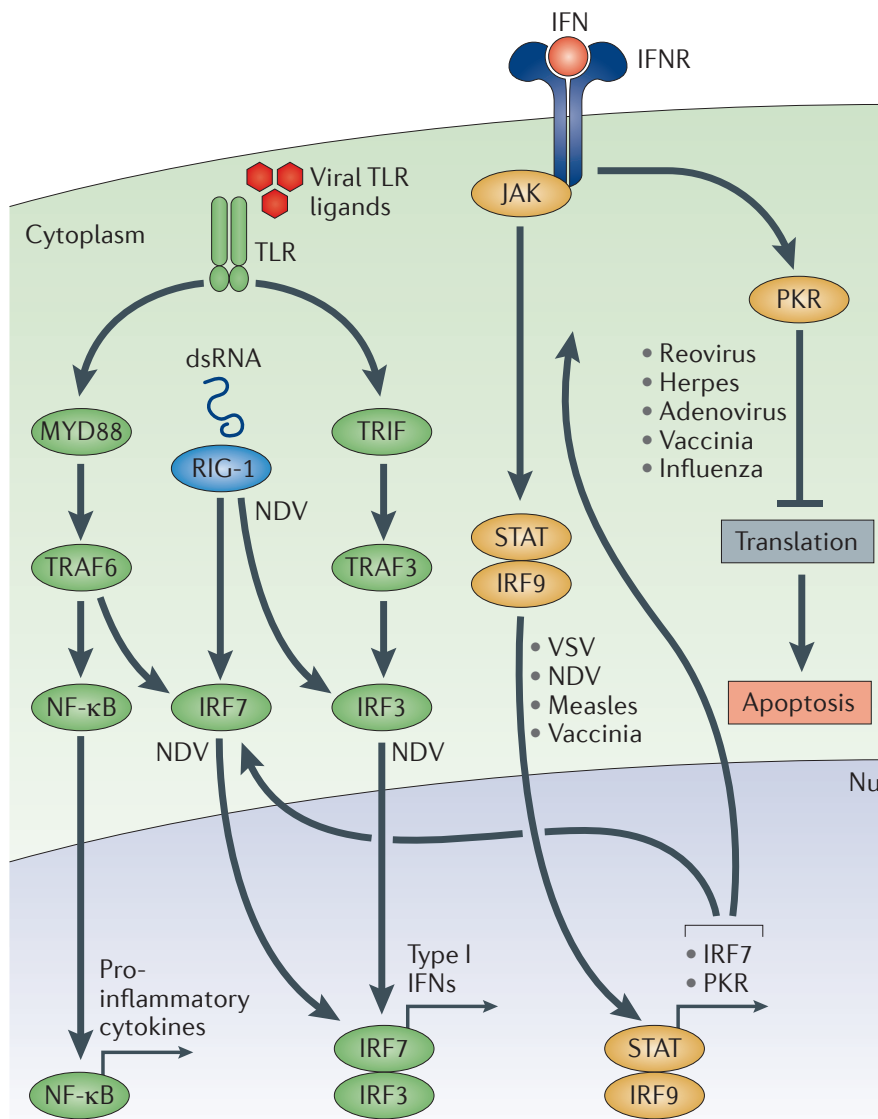

b Cancer cell

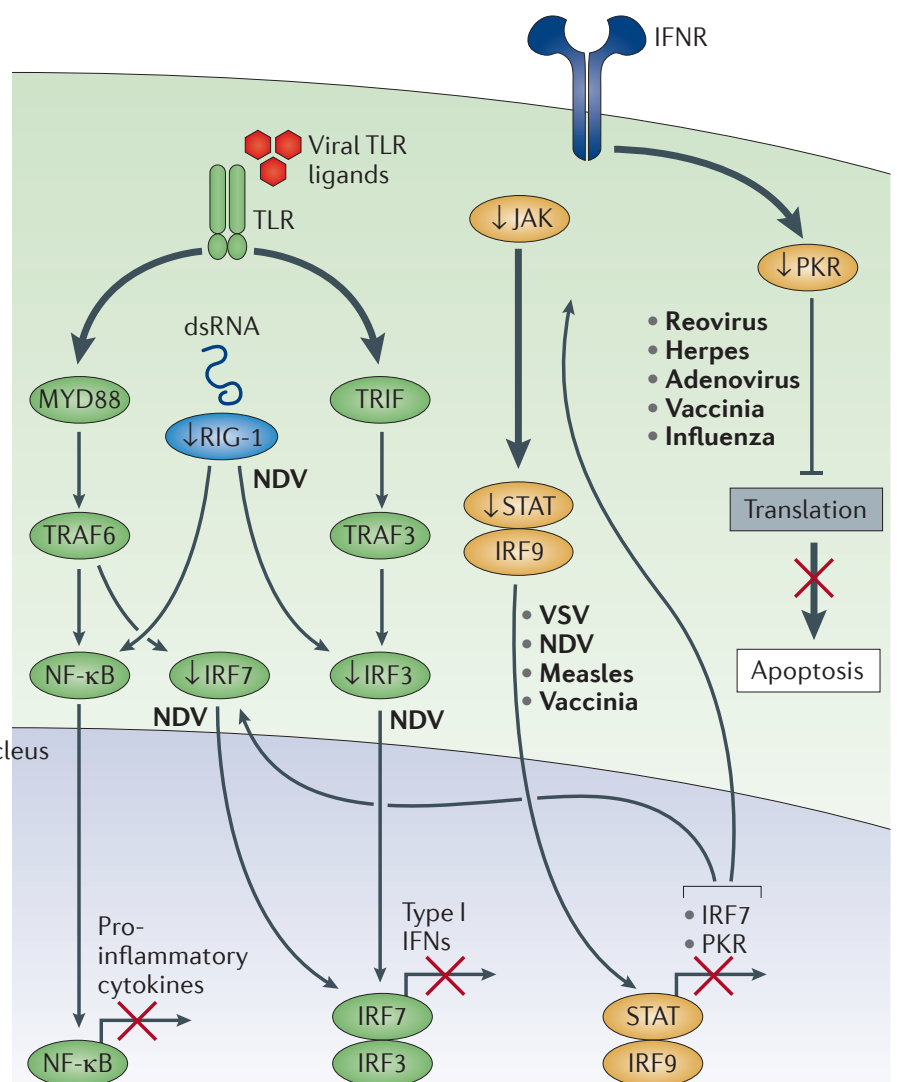

Figure 1 | Oncolytic viruses can exploit cancer immune evasion pathways. a| Following viral infection, most normal cells activate an antiviral pathway that allows to contain viral infections. The antiviral machinery can be triggered by viral pathogen-associated molecular patterns (PAMPs) that activate Toll-like receptors (TLRs) or through the detection of viral nucleic acids by retinoic acid-inducible gene 1 (RIG-1). Once a virus is detected, a signalling cascade through several type I interferon (IFN) elements (Janus kinase (JAK), signal transducer and activator of transcription (STAT), and interferon regulatory factor 9 (IRF9)) results in a programmed transcriptional pathway that limits viral spread and can target infected cells for apoptosis or necrosis. Local IFN production induced by the innate immune response to viral infections may also promote antiviral activity through the IFN receptor (IFNR). TLRs signal via the myeloid differentiation primary response protein MYD88, TIR-domain-containing adapter-inducing IFN $\beta$ (TRIF), IRF7, IRF3 and nuclear factor- $\mathrm{B}$ (NF-kB), inducing the production of pro-inflammatory cytokines and type I IFNs. The type I IFNs signal through the JAK-STAT signalling pathway, resulting in the upregulation of cell cycle regulators, such as protein kinase R (PKR) and IRF7, which limit viral spread by binding to viral particles and triggering type I IFN transcriptional pathways, promoting abortive apoptosis of infected cells and the production of cytokines that alert the immune system to the presence of a viral infection. $\mathbf{b}$ | In cancer cells, however, this process is disrupted. Cancer cells may downregulate key signalling components within the innate signalling pathway, including RIG-1, IRF7, and IRF3 (REF. 1). This limits detection of viral particles by TLR and RIG-1, making cancer cells more susceptible to viral replication. Furthermore, cancer cells may downregulate key components of the type I IFN signalling pathway ${ }^{2-7}$, thereby limiting the pro-apoptotic and cell cycle regulatory effects of type I IFNs. Although data are limited, the figure depicts individual viruses near the factors and/or pathways that are known to promote viral elimination in normal cells (part a) or that support viral replication owing to factor deficiency in cancer cells (part b). dsRNA, double-stranded RNA; NDV, Newcastle disease virus; TRAF, TNF-associated factor; VSV, vesicular stomatitis virus.

Different viruses can also manipulate distinct aberrant signalling factors within tumour cells to block apoptosis, which allows more time for the virus to complete its life cycle. Following viral replication, most oncolytic viruses induce cell death, which can directly eliminate viable tumour cells but also sets the stage for initiating systemic immune responses. Induction of host immune responses can be greatly aided by both the type of cell death and the release of danger signals from virus-infected cells. For example, necrosis or pyroptosis are more immunogenic forms of cell death than apoptosis.

Induction of systemic anti-tumour immunity. The induction of systemic innate and tumour-specific adaptive immune responses appears to be a critical element for tumour eradication with oncolytic viruses. Following oncolytic 
Epitope spreading

A process whereby tissue damage during an initial

immune response against one antigen may lead to the priming of self-reactive $T$ and/or B cells targeting other areas (or epitopes) within the initial antigen or against other antigens.

\section{Neo-antigens}

New antigens, often derived from cell metabolic pathway proteins, and commonly expressed in tumour cells. Neo-antigens may appear as a consequence of epitope spreading following initial immune attack on a tumour cell triggered by an unrelated antigen.

Checkpoint inhibitors Monoclonal antibodies that inhibit negative immune checkpoints. Immune checkpoints control important intracellular signalling pathways in the immune system that either activate or inhibit immune responses. Checkpoint inhibitors have shown significant promise in the treatment of cancer Most notably, blockade of cytotoxic T lymphocyte antigen 4 (CTLA4) and interactions between programmed cell death 1 (PD1) and programmed cell death 1 ligand 1 (PDL1) have had significant impact on the treatment of melanoma, lung cancer, and possibly many other cancers. cell death, tumour cells release tumour-associated antigens that can serve to promote an adaptive immune response that mediates tumour regression at distant tumour sites that are not exposed to virus. They also release viral PAMPS and additional cellular dangerassociated molecular pattern signals (DAMPs; for example, heat shock proteins, high mobility group box 1 (HMGB1) protein, calreticulin, ATP, and uric acid) and cytokines (for example, type I IFNs, tumour necrosis factor- $\alpha$ (TNF $\alpha$ ), IFN $\gamma$, and interleukin-12 (IL-12)), which promote the maturation of antigen-presenting cells (APCs) such as dendritic cells. These activate antigen-specific $\mathrm{CD} 4^{+}$ and $\mathrm{CD}^{+} \mathrm{T}$ cell responses. Once activated, $\mathrm{CD}^{+} \mathrm{T}$ cells can expand into cytotoxic effector cells with the ability to traffic to sites of established tumour growth, where they mediate anti-tumour immunity upon antigen recognition (FIG. 2). However, the natural ability of viruses to induce a host antiviral immune response may result in clearance of the virus through neutralizing antiviral antibodies and/or cytotoxic T-cell-mediated immune responses. The extent to which viral neutralization influences the induction of tumour immunity is complex and can be influenced by many variables, most notably the characteristics of the virus and the tumour microenvironment.

The release of tumour-associated antigens, especially in combination with local cytokine and DAMP release, can be beneficial for inducing innate and adaptive immune responses against cancer cells (FIG. 2). This effect may be especially important for mediating tumour regression at distant tumour sites that are not injected or exposed to virus. Preclinical studies have demonstrated the importance of tumour-specific $\mathrm{CD}^{+} \mathrm{T}$ cells in mediating tumour rejection with oncolytic viruses ${ }^{13}$.

Type I IFNs and DAMPs can also directly activate natural killer (NK) cells, which are part of the innate immune response. NK cells can kill target cells with downregulated major histocompatibility complex (MHC) class I expression, which is a common occurrence in cancer cells ${ }^{14,15}$. The influence of NK cells may depend on both the host species and the characteristics of the virus $^{16,17}$. Furthermore, NK cells may be detrimental to the effectiveness of oncolytic viruses by eliminating virally infected cells ${ }^{18}$. The factors that influence the balance between immune-mediated viral clearance and induction of antitumour immunity are incompletely understood.

Counteracting cancer-mediated immune evasion. Cancer cells have evolved sophisticated strategies for avoiding immune-mediated destruction. For example, tumour cells and the microenvironment can express immuneinhibitory surface receptors that inactivate effector immune cells, and secrete factors - such as IL-10, transforming growth factor- $\beta$ (TGF $\beta$ ) and indoleamine-2,3dioxygenase (IDO) - that facilitate the recruitment of immune-suppressive cells, such as tumour-associated macrophages ${ }^{19}$ and myeloid-derived suppressor cells ${ }^{20}$, to sites of tumour growth ${ }^{21}$. Oncolytic viruses modify this suppressive microenvironment through a variety of mechanisms that alter the cytokine milieu and the type of immune cells within the tumour microenvironment $^{22,23}$. These changes promote immune-mediated tumour cell recognition and eradication, and can trigger tumour-associated antigen and epitope spreading ${ }^{24,25}$. In the presence of danger signals and TLR engagement, the levels of type I IFNs and other inflammatory mediators increase, further potentiating systemic immunity against the cancer.

The killing of cancer cells can result in the release of novel cancer antigens (neo-antigens) that may have been previously hidden to the immune system because of restricted presentation (FIG. 2). This effect was recently reported following the treatment of cancer patients with immunotherapeutic $\mathrm{T}$ cell checkpoint inhibitors ${ }^{26,27}$. Such neo-antigens may be taken up by local APCs in the context of a pro-inflammatory environment, which can trigger an immune response against the neo-antigen. If new $\mathrm{T}$ cell clones are generated, they may be able to circulate and kill antigen-expressing cancer cells, including cancer cells that were not infected by the virus. The immune response has also been associated with a 'immune-associated' bystander effect, in which local release of cytotoxic perforins and granzymes may result in the killing of nearby tumour cells, even in the absence of direct antigen expression ${ }^{28}$. This is distinct from the virus bystander effect (BOX 1), which relates to the replication of the virus inside cancer cells and its spread to previously un-infected cancer cells.

Oncolytic virus biodistribution. Physical barriers that reduce the spread of oncolytic viruses include necrosis, calcification, hypoxia, acidosis, increased proteolytic activity, and a high interstitial pressure ${ }^{29-31}$. Furthermore, tumours are dense with extracellular matrix and are poorly vascularized. The majority of clinical studies with oncolytic viruses (such as adenovirus, poxvirus, HSV-1, measles, and reovirus) have used intratumoural injections to bypass the tumour architectural barriers. However, intratumoural injections are limited to tumours that are physically accessible through clinical palpation or direct imaging. As discussed above, injection of an oncolytic virus into one tumour lesion can induce a systemic anti-tumour response that can overcome physical limitations, as evidenced in the T-VEC OPTIM Phase III clinical trial $^{3}$. Other viruses, such as Seneca Valley Virus, can be delivered intravenously because of natural resistance to haemagglutination, a process resulting in premature viral clearance and reduced delivery to the tumour site following intravenous delivery ${ }^{32}$.

The blood-brain barrier may limit the ability of some viruses (and many other drugs) to reach primary brain tumours and brain metastases. This may be overcome by direct injection into central nervous system (CNS) tumours or through the use of external reservoirs that communicate with sites of brain tumours. Parvovirus naturally crosses the blood-brain barrier, allowing for the delivery of this oncolytic virus via the intravascular route. Parvovirus H-1PV has been used in clinical trials of glioblastoma multiforme $(\mathrm{GBM})^{33}$. However, there have been relatively few studies of oncolytic virus distribution in vivo to evaluate viral penetrance throughout the $\mathrm{CNS}^{33-35}$

Tumour size and heterogeneity can present another barrier to virus biodistribution. Moreover, growtharrested cancer cells in hypoxic environments are less 


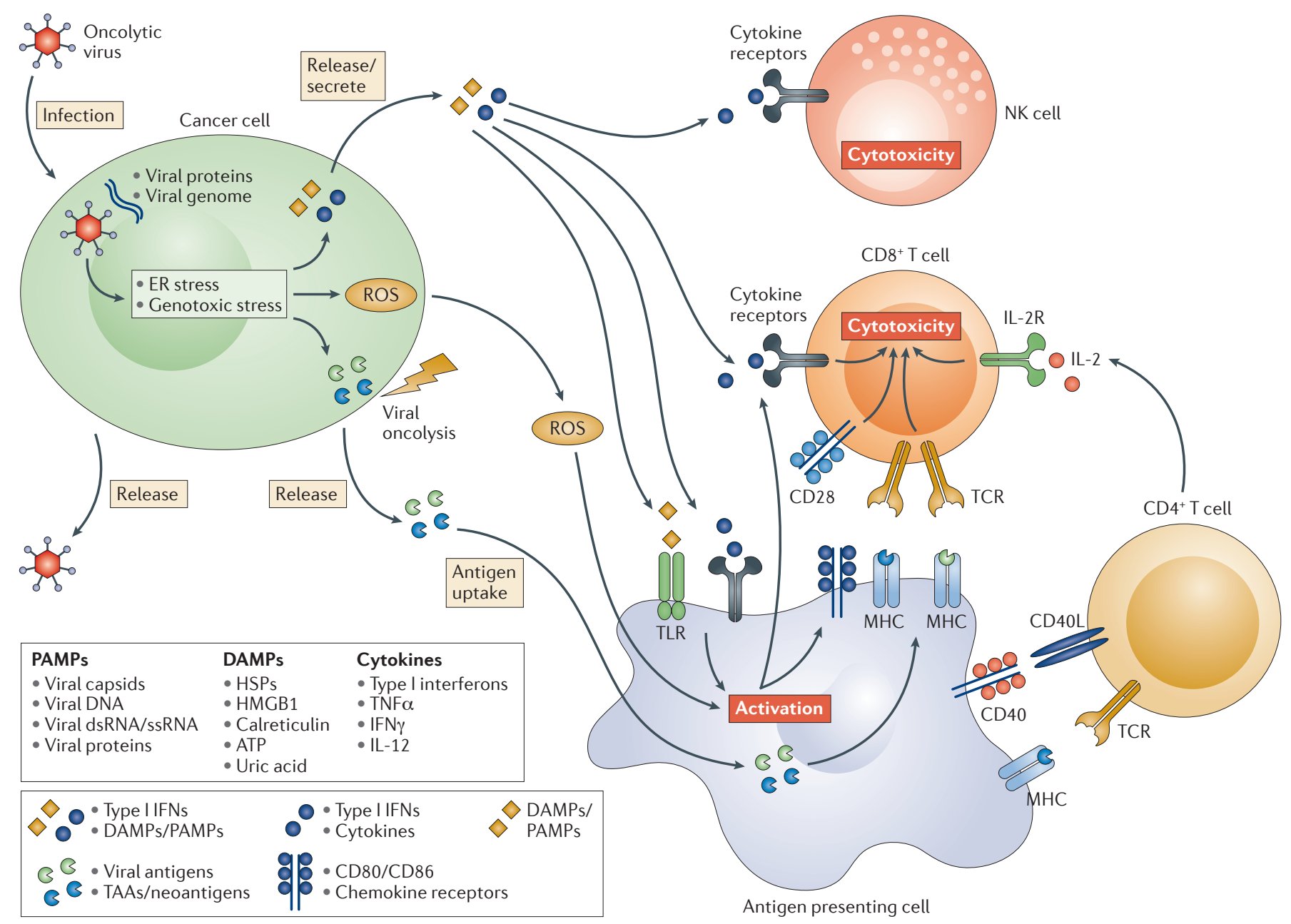

Figure 2 | The induction of local and systemic anti-tumour immunity by oncolytic viruses. The therapeutic efficacy of oncolytic viruses is determined by a combination of direct cancer cell lysis and indirect activation of anti-tumour immune responses. Upon infection with an oncolytic virus, cancer cells initiate an antiviral response that consists of endoplasmic reticulum (ER) and genotoxic stress. This response leads to the upregulation of reactive oxygen species (ROS) and the initiation of antiviral cytokine production. ROS and cytokines, specifically type I interferons (IFNs), are released from the infected cancer cell and stimulate immune cells (antigen presenting cells, CD8 ${ }^{+} \mathrm{T}$ cells, and natural killer (NK) cells). Subsequently, the oncolytic virus causes oncolysis, which releases viral progeny, pathogen-associated molecular patterns (PAMPs), danger-associated molecular pattern signals (DAMPs), and tumour associated antigens (TAAs) including neo-antigens. The release of viral progeny propagates the infection with the oncolytic virus. The PAMPs (consisting of viral particles) and DAMPs (comprising host cell proteins) stimulate the immune system by triggering activating receptors such as Toll-like receptors (TLRs). In the context of the resulting immune-stimulatory environment, TAAs and neo-antigens are released and taken up by antigen presenting cells. Collectively, these events result in the generation of immune responses against virally infected cancer cells, as well as de novo immune responses against TAAs/neo-antigens displayed on un-infected cancer cells. CD40L, CD40 ligand; dsRNA, double-stranded RNA; HMGB1, high mobility group box 1; HSP, heat shock protein; IL-2, interleukin-2; IL-2R, IL-2 receptor; MHC, major histocompatibility complex; ssRNA, single-stranded RNA; TCR, T cell receptor; TNFa, tumour necrosis factor- $\alpha$.

likely to be permissive to infection ${ }^{31,36,37}$. Stromal cells, such as cancer-associated fibroblasts, may be infected by oncolytic viruses but are non-permissive to viral replication. Thus, fibroblasts may act as a decoy reservoir for oncolytic viruses, reducing the delivery of infectious virions to cancer cells ${ }^{38}$. Another mechanism that may limit the overall effectiveness of oncolytic viruses is the susceptibility of cancer cells to apoptosis, which may be induced by viral infection or other factors ${ }^{39}$. If cells undergo apoptosis too rapidly, this will reduce the time for viral replication and propagation and decrease the amount of active virus in the tumour, ultimately limiting the active intratumoural dose.

\section{Development of oncolytic viruses as drugs}

As oncolytic viruses are live viral particles, the overall design of oncolytic virus strategies must consider approaches to tumour cell targeting and attenuating viral pathogenesis, as well as approaches to limit viral immunogenicity while promoting tumour cell killing 


\section{Box 1 | Virus bystander effect}

The optimal method for the administration of oncolytic viruses is not established but most clinical trials have utilized direct injection into established tumours, with some evaluating intravenous delivery. Because oncolytic viruses are capable of replication, only a limited number of tumour cells need to be infected. A significant bystander effect can be anticipated by the local replication of the virus and its release into the surrounding tumour where viral particles can then infect new tumour cells. If the host antiviral immune response does not neutralize the virus, infection may continue to propagate. The inclusion of suicide genes within the oncolytic virus can further enhance the bystander effect and achieve much greater cell killing. However, the contribution of the bystander effect to the overall therapeutic effectiveness of the oncolytic virus needs to be demonstrated in a randomized clinical trial to be more fully evaluated.

and immunogenicity. The flexibility of recombinant engineering has allowed the exploration of a number of strategies to enhance the effectiveness of oncolytic viruses.

Targeting oncolytic viruses to cancer cells. Many of the oncolytic viruses that are currently in the clinic have a natural tropism for cell surface proteins that are aberrantly expressed by cancer cells (FIG. 3). For example, HSV-1 uses the herpesvirus entry mediator (HVEM) and selected nectins for cell entry. These surface receptors are overexpressed on some cancer cells, including melanoma and various carcinomas ${ }^{40}$. Measles virus, specifically the Edmonston strain, utilizes the surface receptor CD46 for cell entry ${ }^{41}$. CD46 normally functions to prevent cell elimination by inactivating the complement pathway of the immune system, and is often overexpressed by cancer cells $s^{42}$. Coxsackievirus can enter cells via intercellular adhesion molecule 1 (ICAM-1; also known as CD54) and decay accelerating factor (DAF; also known as CD55), which can be overexpressed in cancers such as multiple myeloma, melanoma, and breast cancer (although coxsackieviruses may also use nectins for cell entry) ${ }^{43-45}$. Echovirus, a member of the enterovirus family, has increased specificity for ovarian cancer cells because it uses the I domain of integrin $\alpha 2 \beta 1$ for cell entry, which can be overexpressed on these cells ${ }^{46}$. Another member of the enterovirus family, poliovirus, has enhanced specificity for various cancers through its targeting of CD155, a receptor that can potentially impair antitumour NK cell responses and is overexpressed by some cancer cells ${ }^{26}$. Sindbis virus, a member of the Togaviridae family, targets cancer cells that overexpress the $67 \mathrm{kDa}$ laminin receptor, which promotes cancer cell invasion and motility ${ }^{47}$.

Oncolytic viruses can also be engineered to directly target unique cell surface receptors expressed by cancer cells. Examples include the adenovirus Ad5/3- $\Delta 24$, which was modified to bind to integrins that are highly expressed on ovarian cancer cells, and is currently being investigated in clinical trials ${ }^{48,49}$. Other examples of engineered specificities include lentiviruses pseudotyped with the modified E2 glycoprotein from Sindbis virus, which was shown to increase specificity for human melanoma in a mouse xenograft model ${ }^{50}$. Furthermore, measles virus has been engineered to express a single-chain antibody that recognizes carcinoembryonic antigen (CEA), a tumour antigen that is selectively expressed on certain adenocarcinomas ${ }^{51}$.
Exploiting aberrant signalling pathways in cancer. A number of molecular targets promote virus accumulation and replication within tumour cells. For example, tumour cells frequently overexpress the B cell lymphoma (BCL) family of cell survival proteins, which is a hallmark of neoplastic transformation. NDV targets cancer cells overexpressing BCL- $\mathrm{X}_{\mathrm{L}}$, because the overexpression of this protein prevents apoptosis and thus permits the incubation time needed by the virus to multiply and form syncytia required for viral spread ${ }^{52}$.

In addition to the BCL family, the RAS signalling pathway regulates many aspects of carcinogenesis, including resistance to cell death and proliferation ${ }^{53-56}$ (FIG. 4). Reovirus and vaccinia virus show natural selectivity for cancer cells with an over-active RAS signalling pathway. In normal healthy cells, reovirus is able to enter the cell and begin producing viral RNAs, which activates the PKR pathway. Activated PKR, in turn, inhibits protein translation, preventing the production of viral particles and stopping the spread of the virus ${ }^{57}$. However, RAS-transformed cancer cells do not initiate the PKR pathway, rendering cancer cells permissive to viral infection and ultimately cell lysis ${ }^{53}$. An attenuated HSV-1 oncolytic virus, in which the viral genes encoding ICP34.5 and unique short 11 glycoprotein (US11) are deleted, results in preferential lysis of tumour cells compared to normal cells ${ }^{74,79}$. This occurs, in part, because these deletions render HSV-1 unable to block PKR phosphorylation, so it can only replicate in cells with defective PKR signalling ${ }^{87}$. Vaccinia virus, a member of the poxvirus family, depends on epidermal growth factor receptor (EGFR)-induced RAS signalling for viral replication ${ }^{54}$. Poxviruses encode ligands that trigger EGFR signalling, and so cancer cells that overexpress EGFR (and therefore have augmented RAS signalling) are more permissive to vaccinia virus infection ${ }^{54}$.

Cancer cells often have defects in the antiviral mechanism based on type I IFN signalling, providing some viruses with a replicative advantage in cancer cells. These include vaccinia virus ${ }^{54}, \mathrm{NDV}^{58}$, rabbit myxoma virus ${ }^{59}$, mumps $^{60}$, alphaviruses ${ }^{60}$, and vesicular stomatitis virus (VSV) variants ${ }^{61}$.

Type I IFNs are critical for antiviral and antitumour responses in healthy cells not only because they promote immune responses to clear the virus, but also because they reduce cellular proliferation and activate the proapoptotic protein p53 (REF. 62). Multiple types of cancer inactivate this pathway, either by reducing the expression of type I IFNs or by limiting type I IFN signalling via reduced receptor expression or altered downstream signalling (FICS 1,2). Thus, oncolytic viruses have increased specificity for cancer cells and environments in which the type I IFN responses are limited, because in healthy cells viruses are cleared by IFN-mediated responses.

Oncolytic viruses can also be engineered to take advantage of the abnormal signalling pathways in cancer cells by inserting promoters that are more active in cancer cells or that are tissue-restricted. For example, an adenovirus (CV706), in which E1A (an adenoviral protein that inhibits the cell cycle) was placed under the control of the promoter for prostate-specific antigen (PSA), is currently being evaluated in patients with 


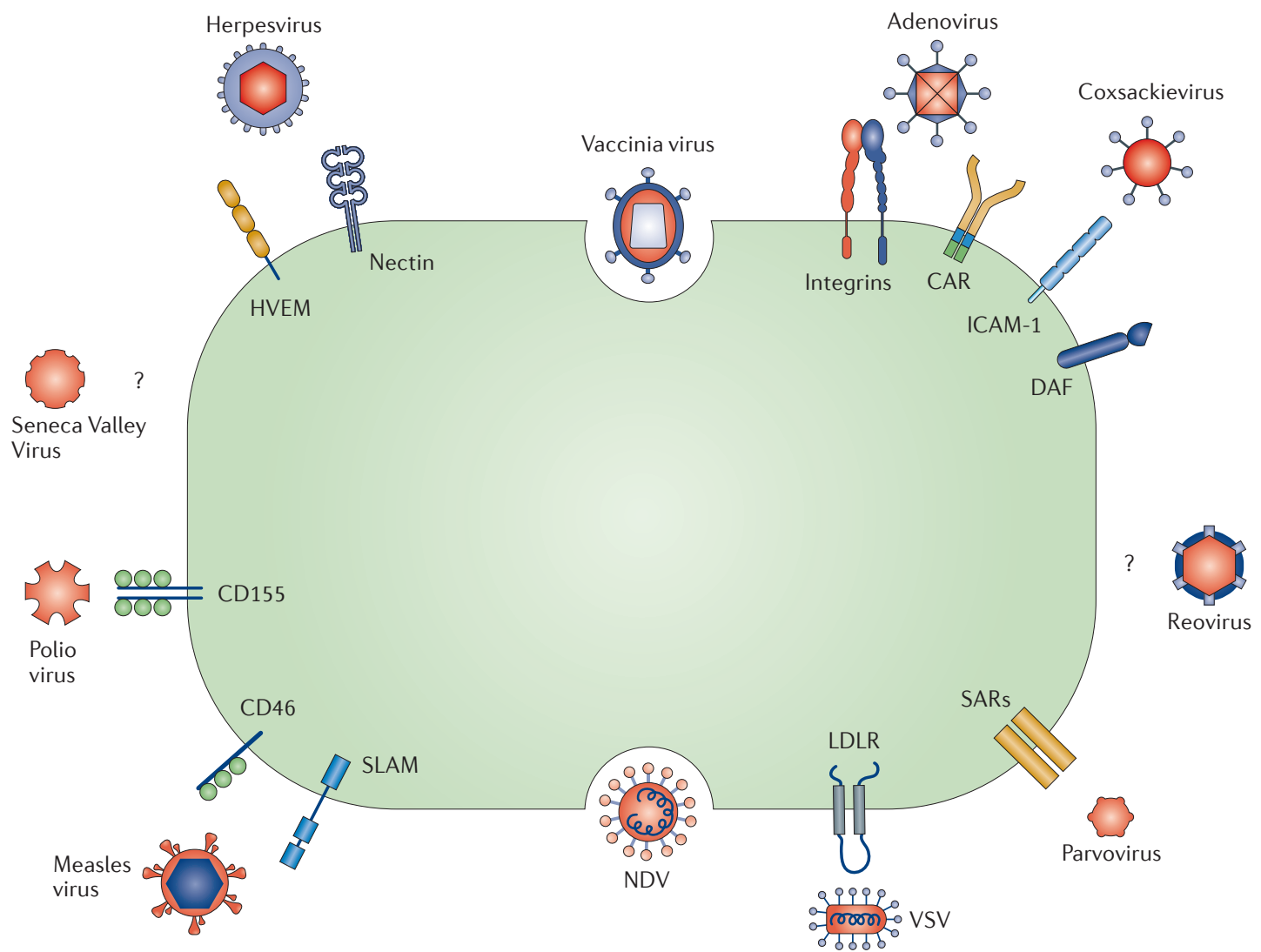

Figure 3 | Mechanisms of viral entry into cancer cells. Oncolytic viruses utilize several mechanisms to enter host cells, including cell surface receptors that are frequently overexpressed on cancer cells. Some viruses are able to via more than one receptor and some receptors can promote the entry of more than one type of virus. Some viruses use endocytosis through membrane fusion and syncytia formation to enter cells. Certain oncolytic viruses are known to preferentially target cancer cells but the cell surface receptor for entry has not been identified. CAR, coxsackievirus-adenovirus receptor; DAF, decay accelerating factor; HVEM1, herpesvirus entry mediator 1; ICAM-1, intercellular adhesion molecule 1; LDLR, low-density lipoprotein receptor; NDV, Newcastle disease virus; SARs, sialic acid receptors; SLAM, signalling lymphocytic activation molecule; VSV, vesicular stomatitis virus (VSV).

prostate cancer ${ }^{63}$. This virus does not produce E1A in healthy cells and these cells undergo apoptosis, thereby restricting viral proliferation in healthy tissue. However, in prostate cancer cells the PSA promoter is highly active and E1A is selectively expressed, resulting in proliferation of the adenovirus and virus-mediated cell lysis. In a similar manner, the oncolytic adenovirus KH901 was engineered to express E1A under the control of the human telomerase reverse transcriptase (TERT) promoter, which is activated in a large number of cancers ${ }^{64}$. Furthermore, two binding sites of the transcription factor E2F1 (a regulator of the cell cycle) were included in the TERT promoter to restrict proliferation to actively dividing cells. Another example is the oncolytic adenovirus CG0070, for which replication is restricted to retinoblastoma ( $\mathrm{Rb}$ )-defective cells (a common mutation in cancer) because E1A expression was placed under the regulation of the E2F1 promoter ${ }^{65}$. In healthy cells, Rb inhibits E2F, thus inhibiting E1A transcription $^{66}$. Furthermore, adenoviruses have been engineered to selectively replicate in hypoxic environments, such as those found inside tumours, by placing the E1A gene under transcriptional regulation by the hypoxia-induced transcription factor HIF-1 $\alpha$; this strategy was shown to be effective in a murine xenograft model of glioma ${ }^{67}$. Several oncolytic adenoviruses, including ONYX-015 and H101, have been designed with a deletion in the gene coding for the protein E1B, which can bind to and inactivate the pro-apoptotic protein p53 (REFS 85,86). Thus, healthy cells that are infected with these viruses can undergo p53 - mediated abortive apoptosis, whereas cancer cells that commonly inactivate p53 remain susceptible to viral infection. Furthermore, these adenoviruses preferentially proliferate in and lyse p53-deficient cancer cells, which account for nearly $50 \%$ of all cancers.

Another approach to improve post-entry tumour specificity has been to encode synthetic miRNA targeting sequences (miRTS) into the $3^{\prime}$ untranslated region (UTR) of the fusion gene of the measles virus. These sequences bind cellular microRNAs (miRNAs) and repress viral replication. Because normal and cancer cells exhibit differential expression of cognate miRNA elements, the engineered oncolytic virus with miRTS can be blocked from replicating in normal cells where specific miRNAs are expressed. Such a construct was 
designed for use in glioma, where it was shown to limit viral replication in neurons that constitutively express high levels of miR-7 while allowing proliferation in glioma cells that frequently downregulate miR-7 (REF. 68).
Attenuating viral pathogenesis. Oncolytic viruses are live viruses that can potentially cause acute toxicity and, in some cases, latent infection and chronic disease. Although the oncolytic activity may be most profound a Healthy cell

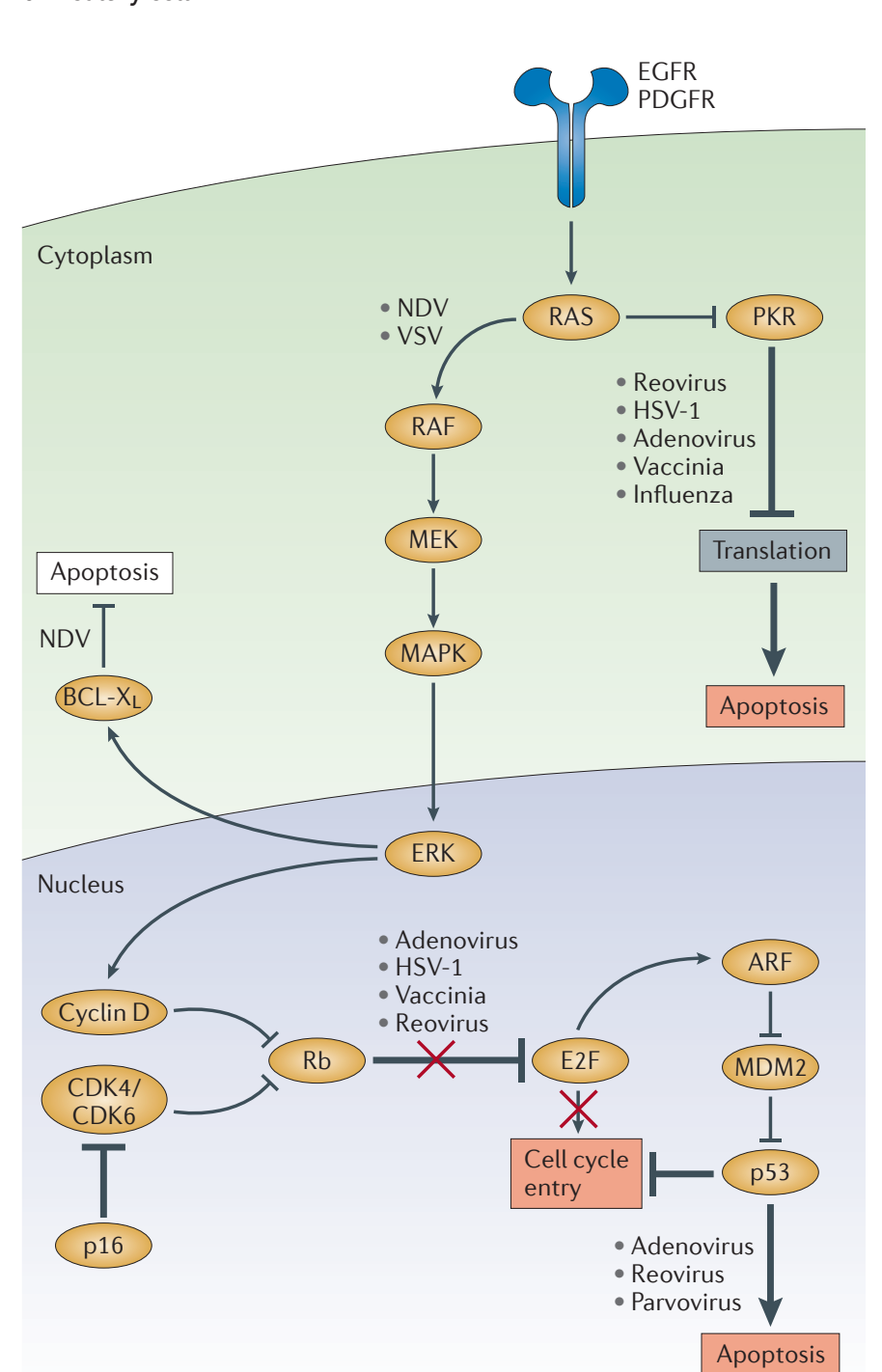

b Cancer cell

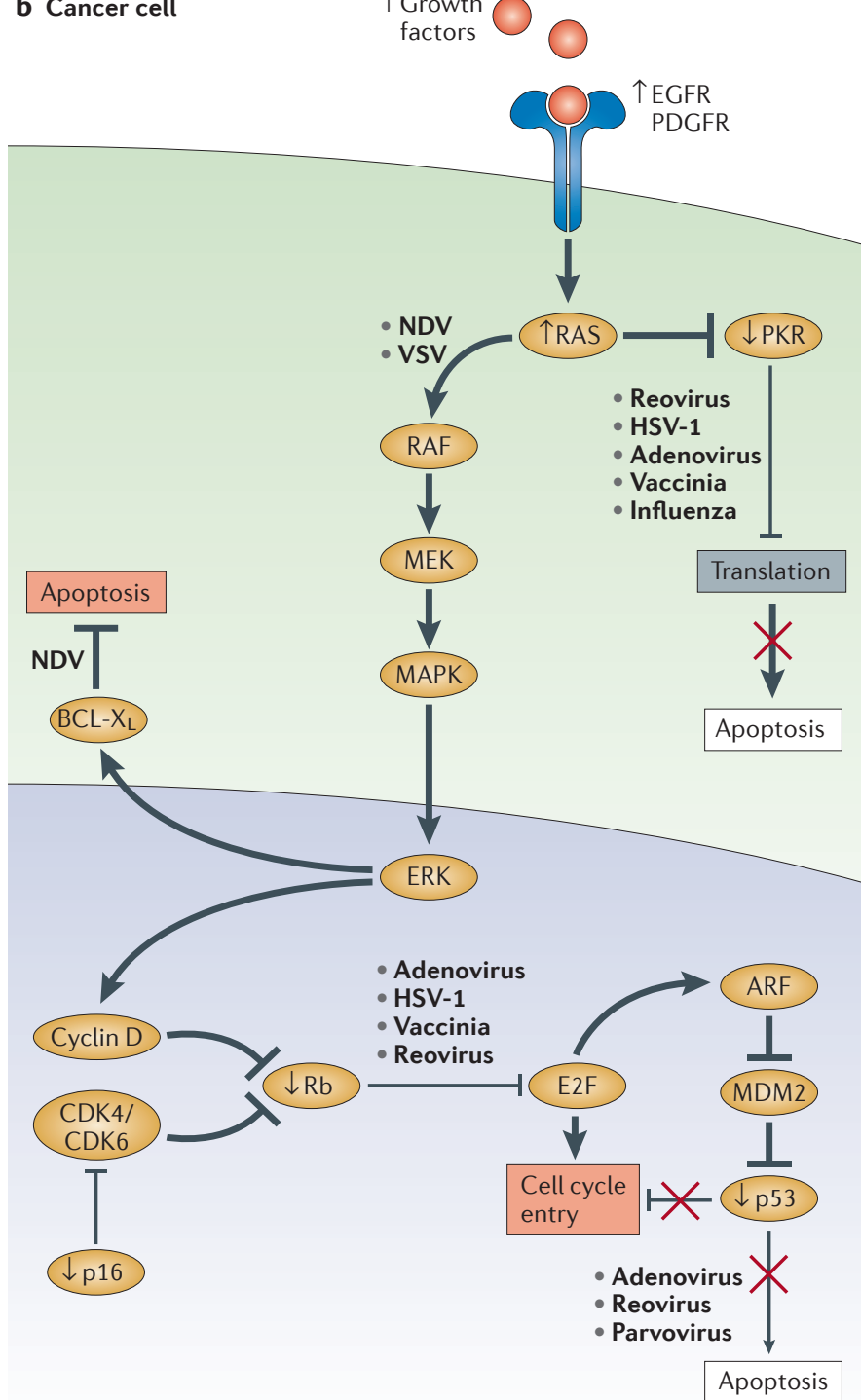

Figure 4 | Oncolytic viruses can target oncogenic pathways. The expression of oncogenes and other aberrant host cell proteins in cancer cells can promote viral replication and oncolytic activity. a | In healthy cells, regulation of cell cycle entry and proliferation is provided by key factors, such as protein kinase $R(P K R), p 16$, retinoblastoma (Rb), and the tumour suppressor $\mathrm{p} 53$. These elements promote abortive apoptosis when the cell cycle is dysregulated. PKR may also help to regulate transcription and induce abortive apoptosis when cells are infected with a virus. $\mathbf{b} \mid$ In cancer cells, cell cycle regulation and cellular proliferation are typically disrupted due to the activity of oncogenes and the loss of tumour suppressor genes. These changes can support viral replication and promote oncolytic virus-induced cell death. For example, activating mutations in the small GTPase RAS increase cell proliferation, which is accompanied by increased protein production. This process can be usurped by oncolytic viruses to replicate more efficiently, as reported for Newcastle disease virus (NDV) and vesicular stomatitis virus (VSV). Furthermore, hyperactive RAS blocks PKR, a process that can facilitate the selective replication of oncolytic viruses (such as reovirus, herpes simplex virus type 1 (HSV-1), adenovirus, vaccinia virus and influenza virus), in RAS-mutant cancer cells. Some viruses, such as adenovirus, reovirus and parvovirus, preferentially target p53-mutant or p53-null cancer cells because healthy cells with intact $\mathrm{p} 53$ undergo abortive apoptosis upon infection. Likewise, aberrant expression of Rb and p16, which regulate cell cycle entry, can render cancer cells susceptible to oncolytic viruses such as adenovirus, HSV-1, vaccinia virus and reovirus. Cancer cells also frequently upregulate the anti-apoptotic protein $B$ cell lymphoma- $X_{L}\left(B C L-X_{L}\right)$. This process confers a selective advantage for oncolytic viruses such as NDV, as it allows more time for viral replication. CDK, cyclin-dependent kinase; EGFR, epidermal growth factor receptor; ERK, extracellular signal-regulated kinase: MAPK, mitogen-activated protein kinase; MEK, MAPK/ERK kinase; PDGFR, platelet-derived growth factor receptor. 
with native viruses, the potential for viral pathogenesis may also be high, resulting in a limited benefit-risk ratio. The potential pathogenicity is highly dependent on the virus, the presence of natural or engineered attenuation factors (for example, virulence, and immune-evasive and latency-promoting genes) and the host immune response. To date, few serious adverse events have been reported in clinical trials but follow-up of patients is often short or incomplete. Most clinically relevant oncolytic viruses utilize attenuated vectors or naturally occurring less virulent variants of particular viruses to prevent acute and longterm toxicity. Examples of the latter are NDV-PV701, a variant of the avian virus NDV, which has been tested in clinical trials against $\mathrm{GBM}^{69}$ (see Supplementary information S1 (table)) and the Edmonston strain of measles virus (commonly used for prophylactic measles vaccination), which was selected for clinical trials in patients with GBM (TABLE 3).

An example of an engineered attenuated oncolytic virus is the HSV-1-based T-VEC. HSV-1 is known to cause neurovirulence and latent infection. Toxicity is mediated by the viral gene product ICP34.5, which counteracts the type I IFN response and antagonizes the PKR signalling pathway within non-dividing cells $s^{56,70-73}$. ICP34.5 is deleted in T-VEC, which means that it should not be able to grow within neurons or mediate latent infection. To date, there have not been any reports of latent infection with T-VEC or other attenuated HSV-1 vectors ${ }^{74}$.

Vaccinia virus has also been attenuated to limit its lytic activity to cancer cells. In non-attenuated vaccinia virus infections, a viral protein termed vaccinia growth factor (VGF) is secreted and acts on the host EGFR to activate the RAS signalling pathway. Such activation promotes cellular proliferation, leading to increased production of thymidine kinase (TK), which helps to promote viral replication. However, attenuation of vaccinia virus through the deletion of VGF makes replication in normal cells difficult and allows viral proliferation only in cells with aberrant EGFR-RAS signalling, as frequently found in cancer cells $s^{64,75}$. Another component that is necessary for the infection of healthy cells is the vaccinia protein B18R, which blocks type I IFN signalling ${ }^{76,77}$. By attenuating vaccinia virus through the deletion of B18R, healthy cells become susceptible to the type I IFN antiviral response and the infection is limited. However, cancer cells commonly disrupt the type I IFN pathway to evade the immune system, and thus they are susceptible to infection and subsequent lysis. Furthermore, deletion of $B 18 R$ may increase the activity of oncolytic vaccinia virus because type I IFNs produced as a result of vaccinia infection of the tumour are not blocked by $\mathrm{B} 18 \mathrm{R}^{78}$.

In contrast to deleting pathogenic viral genes, the expression of virulence genes can also be restricted to tumour tissues by incorporating promoters that regulate the expression of virulence genes. As described above, adenoviruses have been engineered with E1A and E1B expression limited to tumour cells through transcriptional regulation by tumour-specific promoters. Although reversion to a native virus through natural homologous recombination remains a theoretical concern, there is no evidence that this happens in the clinic.
Augmenting anti-tumour immunity. The process of tumour clearance by immune stimulation is critical to the anti-tumour activity of oncolytic viruses, and several strategies have been devised to engineer oncolytic viruses so they can more effectively stimulate anti-tumour immune responses. For example, anti-tumour immune responses can be augmented by viral expression of proinflammatory cytokines and/or T cell co-stimulatory molecules. This strategy has been well described for viruses such as HSV-1, adenovirus, and vaccinia virus. Studies of HSV-1 in mice demonstrated rejection of contralateral, un-injected flank tumours only when GM-CSF, a cytokine that promotes dendritic cell accumulation and maturation, was engineered into the viral genome $^{74,79}$. GM-CSF is thought to improve tumour antigen presentation and stimulate robust $\mathrm{T}$ cell responses. GM-CSF has been included in $\mathrm{T}^{-V_{E C}}{ }^{80}$, the adenoviruses CG0070 and CGTG-102 (REF. 81), and the vaccinia virus JX-594 (REF. 82).

An adenovirus that expresses the heat shock protein HSP70 in cancer cells, which increases chaperoning of proteins to proteases that enhance protein degradation and processing, has been used to increase tumour antigen presentation ${ }^{83}$. This approach may also have a role in epitope spreading as APCs preferentially take up peptides bound to HSP70.

For HSV-1-based oncolytic viruses such as T-VEC, strategies have also been devised to enhance immune responses by deleting the HSV-1 protein ICP47. ICP47 blocks the function of TAP (transporter associated with antigen processing) and thus prevents infected cells from presenting antigen to $\mathrm{CD} 8^{+} \mathrm{T}$ cells ${ }^{84}$.

Some cancers, such as glioblastoma, are inherently resistant to apoptosis, even when infected with viruses, owing to dysregulation of apoptotic pathways. An oncolytic parvovirus $\mathrm{H}-1 \mathrm{PV}$, however, was shown to kill glioma cells by activating the immunogenic cathepsinmediated death pathway ${ }^{88}$. Furthermore, parvovirus can also induce an immune response against GBM, potentially through a bystander effect involving an increase in inflammatory cytokines (such as IFN $\gamma$ ) and tumour antigen release $\mathrm{e}^{89,90}$.

Enhancing lytic activity. The inclusion of 'suicide genes' (genes that render cells more sensitive to apoptosis or therapy with other drugs) into oncolytic viruses can enhance their ability to directly kill cancer cells $s^{93-95}$. For example, the pro-apoptotic molecules TNF-related apoptosis-inducing ligand (TRAIL) or TNFa have been included in viral constructs to enhance cell death and trigger an immune response $\mathrm{e}^{91,92}$.

Using tumour-enriched/tissue-specific promoters to preferentially express suicide genes in cancer cells has had success in limiting side effects and improving the therapeutic effectiveness of oncolytic viruses in several preclinical models. For example, the adenovirus Ad-OC-HSV-TK, which is in development for the treatment of bone tumours, encodes the gene for HSV-1 TK driven by the osteocalcin promoter ${ }^{96}$. HSV-1 TK converts thymidine analogues (such as ganciclovir) into monophosphates, which get incorporated into the DNA 


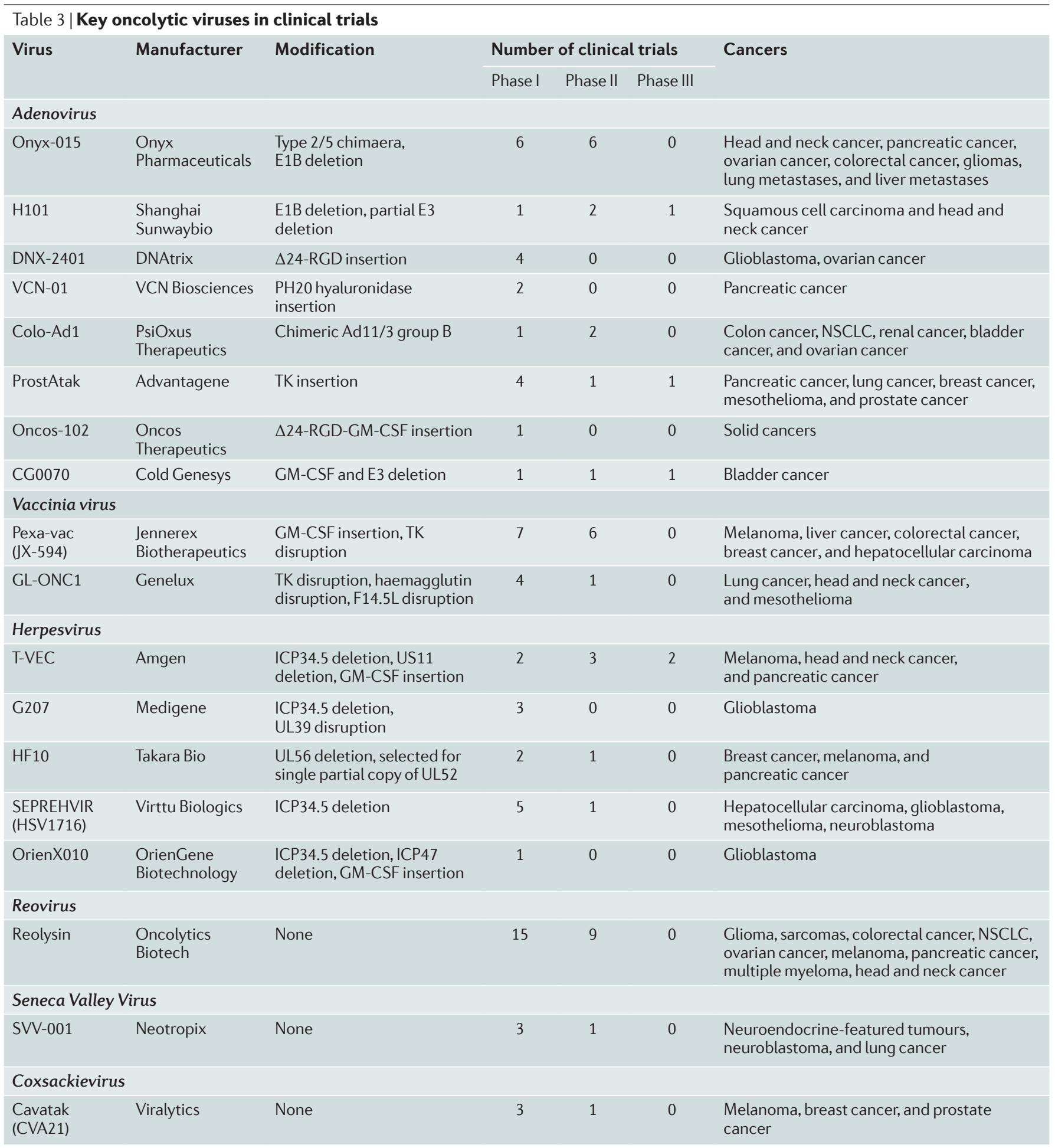

GM-CSF, granulocyte-macrophage colony-stimulating factor, NSCLC, non-small-cell lung cancer; RGD, Arg-Gly-Asp motif; TK, thymidine kinase; US11, unique short 11 glycoprotein.

of replicating cells, resulting in the termination of DNA synthesis and, ultimately, cell death ${ }^{97}$. In this system, the expression of TK is restricted to cells with an active osteocalcin promoter, and this increases their susceptibility to treatment with the thymidine analogue ganciclovir. However, as ganciclovir can block viral replication, this approach may inhibit oncolytic virus activity ${ }^{98,99}$.
Two other suicide genes that have been tested are bacterial cytosine deaminase (CD) and adenovirus death protein $(\mathrm{ADP})^{100,101}$. CD can transform 5-fluorocytosine into 5-fluorouracil (5-FU), which is cytotoxic. ADP is a nuclear membrane glycoprotein that is required in the late stages of adenovirus infection for efficient cell lysis and release of viral particles. Enhanced 
lytic activity was reported for an oncolytic adenovirus that had the adp gene inserted into the adenoviral E3 locus, resulting in ADP overexpression ${ }^{101}$.

Limiting antiviral immune responses. Although immune stimulation is critical to the anti-tumour activity of oncolytic viruses, this effect is balanced by the potentially rapid clearance of the virus by antiviral immunity. Moreover, humans are naturally (or artificially through vaccination) exposed to oncolytic viruses and may therefore have pre-existing neutralizing antibodies or cellular immunity against some oncolytic viruses.

One strategy to limit virus neutralization is to utilize alternative virus serotypes. Multiple serotypes exist for both adenoviruses and VSV, which allows for serotype switching between injections to prevent antibody neutralization. Although measles virus does not naturally switch serotypes, measles viruses that are engineered to mimic serotype switching have been shown to limit neutralizing antibody titres against the virus ${ }^{102}$. Other strategies to overcome viral clearance include PEGylation (covalent conjugation with polyethylene glycol) of the viral coat (VSV and adenovirus) and polymer coating (adenovirus) to prevent antibody binding and neutralization ${ }^{103-105}$. Polymer coating of adenovirus reduces targeting by covering viral proteins required for cell entry, but simultaneously increases circulation time. Investigators have also been able to protect oncolytic viruses from antibodies by using cell carriers (such as mesenchymal stem cells) are infected ex vivo and then transferred back into a host for trafficking to tumour sites $^{106,107}$.

HSV-1-specific T cell responses may be dampened through the expression of the viral US11 gene product, which blocks antigen presentation, thereby limiting detection by $\mathrm{T}$ cells and prolonging viral infection ${ }^{108}$.

In addition to modifying the virus, attempts have been made to suppress the host immune system for example, through pretreatment with cyclophosphamide. This strategy was shown to enhance oncolytic efficacy of HSV- in a glioma model ${ }^{109}$.

Enhancing virus bioavailability. In addition to strategies that avoid viral neutralization, as described above, a number of preclinical studies have investigated other methods for enhancing oncolytic virus bioavailability. For example, treatment with oncolytic viruses has been combined with vasoactive or vaso-normalizing treatments such as histamine ${ }^{110}$, nitroglycerin ${ }^{110}$, local hyperthermia ${ }^{111}$, low-dose paclitaxel $^{112}$, bevacizumab ${ }^{113}$, and bradikynin ${ }^{16}$ to improve viral delivery.

Viral penetrance can also be enhanced by pretreatment of the tumour microenvironment with proteolytic enzymes (that is, hyaluronidase or collagenase) that can break down the sieve-like barrier posed by the extracellular matrix $(\mathrm{ECM})^{114,115}$. Oncolytic viruses have also been engineered to express ECM-degrading enzymes such as hyaluronidase, which was shown to increase tumour dissemination and therapeutic activity in a melanoma xenograft model ${ }^{116}$.
Furthermore, oncolytic viruses are being selected and/or engineered to overcome the physical barriers of the interstitial pressure and the acidic and hypoxic environment present in tumours by spreading between cancer cells following the induction of cell fusion. This process protects the virus from the physical limitations associated with the need for extracellular propagation and limiting the infection to the intracellular space. For example, NDV, coronavirus, orthomyxovirus and paramyxovirus use fusogenic membrane glycoproteins (FMGs) to propagate viral infection between cells. Furthermore, VSV has been engineered to express the NDV fusogenic mutant F glycoprotein ${ }^{117}$ and Sindbis virus has been engineered to express the hyperfusogenic envelope glycoprotein of gibbon ape leukaemia virus to convey fusogenic-based viral propagation ${ }^{118}$. Measles virus has been engineered to activate fusogenic membrane glycoprotein F in the context of high levels of matrix metalloproteinases (MMPs), as present in tumours, rather than through its normal regulation by furin ${ }^{119}$.

\section{Oncolytic viruses in clinical development}

There are numerous oncolytic viruses in clinical development (see Supplementary information S1 (table)), most of which are in early-phase clinical trials. Here, we restrict detailed discussion to viruses that are currently in Phase III development. At present, adenoviruses and herpesviruses have been the most widely evaluated in the clinic.

Herpes simplex viruses. HSV-1 is a member of the alphaherpesvirus family, which includes the varicella species. HSV-1 is a double-stranded DNA virus with a large genome (152kb), in which $\sim 30 \mathrm{~kb}$ encode genes that are not essential for viral infection. HSV-1 replication occurs in the nucleus; however, HSV-1 does not cause insertional mutagenesis. These properties make HSV-1 an attractive candidate for oncolytic virus development. However, HSV-1 is a major human pathogen that causes skin lesions and rashes, moreover it can infect peripheral nerves and enter a latent stage. HSV-1 can infect many types of cells, including epithelial cells which it enters through viral surface glycoproteins, immune cells through HVEM, and neurons through surface nectins (nectin 1 and nectin 2).

T-VEC is the most commercially advanced HSV-1based oncolytic virus. In order to reduce pathogenesis while enhancing selective tumour cell infectivity, $\mathrm{T}$-VEC contains deletions of the neurovirulence gene ICP34.5 and the inhibitor of antigen presentation ICP47 (REFS 74,79). As discussed above, ICP34.5 is critical for blocking the host antiviral PKR-IFN response and required for HSV-1 infectivity of neurons ${ }^{120}$. In T-VEC, two ICP34.5 genes are deleted and this improves cancer cell selectivity and prevents infection of neurons, thereby significantly reducing the overall pathogenesis of HSV-1. ICP47 deletion results in the presentation of viral antigens by both healthy and cancer cells, which leads to the containment of the infection in healthy tissues and immune-mediated destruction of cancer cells that selectively propagate oncolytic HSV-1. The deletion of ICP47 
also induces the early activation of the US11 promoter. HSV-1 US11 blocks PKR phosphorylation, and thereby prevents cancer cells from undergoing abortive apoptosis when infected ${ }^{87}$. Thus, expression of HSV-1 US11 earlier in the viral infection cycle increases oncolytic therapeutic activity. Finally, the gene encoding GM-CSF has been engineered into the viral genome in place of the ICP34.5 genes to improve the induction of antitumour immunity.

In preclinical studies, T-VEC demonstrated potent lytic effects against several tumour cell lines, most notably melanoma and pancreatic cancer cells ${ }^{79}$. T-VEC has been evaluated in advanced clinical trials for patients with melanoma, pancreatic cancer, head and neck tumours, and in 14 patients with breast cancer in a Phase I clinical trial.

In a multi-institutional Phase II clinical trial, patients with unresectable stage IIIC and IV melanoma were treated with an initial dose of $10^{6} \mathrm{pfu}$ (plaque forming units) per $\mathrm{ml} \mathrm{T}$-VEC given by intratumoural injection to allow for seroconversion, and followed 3 weeks later by $10^{8}$ pfu per ml given every 2 weeks thereafter until maximal clinical response, unacceptable toxicity or disease progression ${ }^{79,121}$. In this study, 50 participants were enrolled and an objective response rate of $26 \%$ was reported with generally low-grade constitutional toxicity including fever, fatigue and local injection site reactions. T-VEC was then evaluated in a prospective, randomized clinical Phase III trial in 436 patients with unresectable stage IIIB, IIIC or IV melanoma ${ }^{122}$. The trial met its primary end point, with a durable response rate (defined as an objective response beginning within 12 months of treatment and lasting for at least 6 months) of $16.3 \%$ in patients receiving T-VEC compared to $2.1 \%$ in patients given GM-CSF (odds ratio $=8.9 ; P<0.0001$ ) . This included responses in all lesions, injected and non-injected. An objective response rate of $26.4 \%$ was reported (compared to 5.7\% for GM-CSF-treated participants), with $10.9 \%$ achieving a complete response. In addition, patients given T-VEC had an improved median overall survival (23.3 months versus 18.9 months for GM-CSF-treated patients), although the study was not powered for survival. An especially strong effect was seen in patients with stage III and IV M1a tumours, suggesting that T-VEC might be particularly well suited for the treatment of early-stage patients with less extensive visceral disease. Treatment was well tolerated, with the majority of adverse events related to fever, chills, nausea, fatigue and local injection site reactions. Based on these results, an FDA advisery panel voted to approve T-VEC, and a final ruling is pending.

T-VEC is also being studied in combination with the immunotherapeutics ipilimumab and pembrolizumab in patients with melanoma (BOX 2). These combinations may be more appropriate for patients with more advanced and heavily pretreated disease. Preliminary data from a Phase Ib clinical trial of T-VEC and ipilimumab, a monoclonal antibody that blocks the cytotoxic T lymphocyte antigen 4 (CTLA4), a T cell checkpoint inhibitor, have reported response rates of $50 \%$, with a $22 \%$ complete response rate ${ }^{123}$. These results are now being evaluated in a randomized clinical trial.
Adenoviruses. Adenovirus is a naked (non-enveloped), double-stranded DNA virus with a linear genome of $\sim 35 \mathrm{~Kb}$ encapsulated by an icosahedral capsid with a virion diameter that ranges from 70 to $90 \mathrm{~nm}$. As it has a large genome, long DNA sequences can be incorporated, thus permitting multiple engineered modifications. Adenoviruses commonly infect both humans and animals and can be transmitted through aerosols and direct contact. This results in the majority of the population being seropositive for adenovirus exposure. Although asymptomatic in immune-competent hosts, adenoviral infections can cause disease in newborns and immunocompromised patients. Adenovirus enters the cell using the coxsackie-adenovirus receptor (CAR). Upon cell entry, adenovirus traffics to the nucleus where it expresses adenoviral early genes (encoding E1A and E1B), which are necessary for viral propagation. Specifically, E1A and E1B target the tumour suppressors p53 and retinoblastoma-associated protein (pRb) to promote cell cycle entry. However, in healthy cells the targeting of host cell cycle regulators p53 and pRb by the adenoviral E1A and E1B proteins results in abortive apoptosis and clearance of the virus ${ }^{124,125}$. The ability to easily attenuate viral pathogenicity and encode large foreign transgenes makes adenovirus an attractive vector for clinical development.

There are 57 serotypes of adenovirus, which are classified into categories A-G based on the viral properties of agglutination and oncogenic potential in rodent models. Group $\mathrm{C}$ adenoviruses are non-oncogenic and, in particular, serotypes 2 and 5 have been evaluated as potential oncolytic agents. Early results from clinical trials carried out in the late 1950s in cervical cancer demonstrated limited therapeutic activity with live replicating adenovirus, which may be explained, in part, by the limited expression of CAR on cancer cells ${ }^{126}$. In a recent report, adeno-associated virus (AAV) contamination of an adenovirus preparation resulted in enhanced oncolytic activity by adenovirus, which may be one strategy for improving therapeutic activity ${ }^{127}$. To date, clinical trials have shown that oncolytic adenovirus therapy results in few adverse events, demonstrating a favourable safety profile.

The adenoviral genome is relatively easy to modify, and transgenes of up to $10 \mathrm{~kb}$ can be inserted without disrupting viral infection. Therefore, adenoviruses have been engineered to target surface receptors that are more universally upregulated on cancer cells. This concept is being clinically evaluated in ovarian cancer, using a modified Ad5/3- $\Delta 24$ virus $^{128}$. Because ovarian cancer has a variable expression pattern of CAR, the Ad5/3- $\Delta 24$ virus capsid was modified to incorporate an RGD (ArgGly-Asp) motif into the HI loop of the fibre knob (a portion of the outer layer of adenovirus capsids $)^{48}$. This modification permits cell entry through the binding of adenovirus to other receptors, including $\alpha \mathrm{v} \beta 3$ and $\alpha \mathrm{v} \beta 5$ integrins ${ }^{129}$, which are highly expressed on ovarian cancer cells $^{49}$. DNX-2401, an adenovirus with this RGD motif, has entered Phase I clinical trials in glioma. To further improve targeting of adenoviruses, mosaics (combined serotypes) are being developed. Specifically, the 


\section{Box 2 | Approaches to combination therapy}

Oncolytic viruses have demonstrated very tolerable safety profiles, and their ability to modulate the tumour microenvironment provides a rational strategy for combination treatment with other cancer agents to improve therapeutic responses. Oncolytic viruses can easily be combined with a wide range of therapies, including surgical resection, chemotherapy, radiation therapy, hormonal therapy, targeted therapies, and immunotherapy. Indeed, adenovirus, reovirus, herpes simplex virus type 1 (HSV-1) and parvovirus have been clinically evaluated in combination with surgical resection, chemotherapy, and radiation therapy ${ }^{176-178}$. The rationale for these combinations was based on the expectation that oncolytic viruses would be unaffected by these therapies and able to target residual cancer following standard-of-care treatment. This concept has also been applied with adenoviruses in combination with hormonal therapy in prostate cancer ${ }^{179}$.

Perhaps the most promising strategy is to combine oncolytic viruses with T cell checkpoint inhibitors. The recent advances in the development of checkpoint inhibitors suggest that these are potent agents with activity in a wide range of cancers. Studies suggest that patients with tumours expressing high levels of programmed cell death 1 ligand 1 (PDL1) may have an improved response to T cell checkpoint inhibitors ${ }^{180}$. As oncolytic viruses often induce interferon (IFN) release in the local tumour microenvironment, and IFN is known to upregulate PDL1 expression on tumour cells, this combination is especially interesting ${ }^{181}$. In fact, preclinical models have validated this approach and several clinical trials are under way ${ }^{182}$. Preliminary reports from a Phase Ib trial of T-VEC and ipilimumab support an added therapeutic benefit of combination therapy ${ }^{123}$. adenovirus serotype 35 (Ad35) preferentially uses CD46 rather than CAR for cell entry ${ }^{130}$. CD46 expression prevents normal cells from being recognized by complement and subsequently eliminated. Cancer cells have been shown to overexpress $\mathrm{CD} 46$ and subvert recognition in this manner. Therefore, a mosaic oncolytic adenovirus has been engineered with the CAR-binding sequence of Ad5 replaced with the CD46-binding sequence of Ad35 (REF. 131).

A number of oncolytic adenoviruses in clinical development were designed to take advantage of aberrant signalling pathways in cancer cells. Examples are the adenovirus with a PSA-driven E1A that is being tested in prostate cancer $^{63}$, adenoviruses engineered to use the TERT promoter and the E2F1 promoter to regulate E1A expression ${ }^{64}$, and the adenoviruses ONYX-015 and $\mathrm{H} 101$, which have a deletion in the portion of E1B that inactivates p53 (REFS 34,118).

The ONYX-015 virus was evaluated in several clinical cancer trials but limited responses were reported, and clinical development was halted in 2003 (REF. 132). There may be renewed interest in this agent given the improved understanding of oncolytic viruses and the potential for combination therapy regimens with other anticancer agents.

H101 is an E1B-deleted adenovirus that was approved for the treatment of nasopharyngeal cancer in China. H101 was evaluated in a randomized Phase III clinical trial in 160 participants with advanced squamous cell carcinomas of the head and neck or oesophagus ${ }^{133}$. The patients were randomized to chemotherapy (cisplatin and 5-FU for chemotherapy-naive patients, or adriamycin and 5-FU for patients who had received prior platinum chemotherapy) with or without the addition of H101 $\left(5 \times 10^{11}\right.$ to $1.5 \times 10^{12}$ viral particles per day by intratumoural injection) for 5 consecutive days every 3 weeks.
A total of 123 participants completed treatment and were evaluable for response. Patients who were treated with cisplatin/5-FU and H101 had a 78.8\% response rate, compared to $39.6 \%$ in the cisplatin/5-FU-only cohort. Patients who received the adriamycin/5-FU and H101 virus and the adriamycin/5-FU -only group both had a $50 \%$ response rate but the number of participants in these groups was small $(n=18)$. There was a significant difference in response rate between all patients who received H101 compared to patients treated with chemotherapy alone. The main adverse events reported included fever, injection site reactions and flu-like symptoms. Based on these results, the Chinese regulatory agency approved H101 for the treatment of nasopharyngeal carcinoma in combination with chemotherapy.

Vaccinia virus. Vaccinia virus is a member of the poxvirus family and has a large dsDNA genome $(\sim 190 \mathrm{~kb})$. As vaccinia virus replicates entirely in the cytoplasm of infected cells, concerns regarding the potential for insertional mutagenesis are eliminated. Vaccinia can also infect a wide range of cells and is highly tropic for tumour cells ${ }^{54}$. It is thought to enter host cells by an endocytic process through the cell membrane. These features all make vaccinia virus an attractive vector for oncolytic virus development. Furthermore, vaccinia infections are relatively innocuous in immunocompetent people, although they can result in systemic illness in immunocompromised patients. Vaccinia infection induces potent cellular and humoral immune responses ${ }^{134-136}$. The potential of vaccinia virus to generate potent immunity has been well demonstrated via the use of an attenuated version of vaccinia virus to vaccinate against smallpox, which has led to the successful eradication of the disease.

Vaccinia virus has been attenuated for both its use in vaccination and as an oncolytic agent. Specifically, viral TK, vaccinia growth factor (VGF), and vaccinia type I IFN-binding protein (B18R) have been modified to increase cancer cell selectivity and lysis ${ }^{77,137}$. Based on the importance of the immune response and the ability of the vaccinia viral genome to accept large transgenes $(25 \mathrm{~kb})$, vaccinia virus has been engineered to express tumour antigens, $\mathrm{T}$ cell co-stimulatory molecules, and inflammatory cytokines. Specifically, the expression of tumour antigens PSA, CEA or mucin 1 (MUC1) by vaccinia virus results in the presentation of the tumour antigen in the context of a potent antiviral response, which generates an enhanced antigen-specific antitumour immune response ${ }^{137,138}$. Furthermore, vaccinia virus expression of the co-stimulatory molecule B7-1 by itself or in combination with ICAM-1 and lymphocyte function-associated antigen 3 (LFA3) (the combination is termed TRICOM) provides the co-stimulatory signalling needed by antitumour $\mathrm{CD} 8^{+} \mathrm{T}$ cells and has shown clinical activity with an acceptable safety profile in early-phase clinical trials ${ }^{77}$. Vaccinia vectors encoding tumour antigens and co-stimulatory molecules have been used in clinical development as systemic vaccines, but few studies have focused on using these vectors as oncolytic agents. 
A vaccinia virus encoding the $\mathrm{B} 7.1 \mathrm{~T}$ cell co-stimulatory molecule (rV-B7.1) was tested in a Phase I clinical trial in patients with advanced melanoma. The virus was found to be safe, patients developed melanoma-specific $\mathrm{T}$ cell responses, and 3 out of 12 patients had evidence of tumour regression including one complete response $\mathrm{e}^{139}$. A vaccinia virus encoding TRICOM (rV-TRICOM) was also evaluated in a Phase I melanoma trial with a $30.7 \%$ response rate ${ }^{140}$. In these studies, adverse events were generally mild, consisting of flu-like symptoms and local injection site reactions, with several patients developing autoimmune vitiligo. However, further clinical development has been on hold.

A vaccinia virus encoding GM-CSF (JX-594) has also entered clinical trials ${ }^{82,141,142}$. In a randomized dosefinding study, 30 patients with advanced hepatocellular carcinoma received a low-dose $\left(10^{8} \mathrm{pfu} ; n=14\right)$ or highdose $\left(10^{9} \mathrm{pfu} ; n=16\right)$ intra-tumoural injections into 5 or fewer lesions on days 1, 15 and 29. Viral replication and GM-CSF expression was noted before therapeutic responses. Overall, a 15\% objective response rate, including responses in distant, un-injected lesions, was reported by modified RECIST criteria (Response Evaluation Criteria In Solid Tumours) and was similar at both doses. Median survival, however, was higher in patients who received the high-dose of virus (14.1 months) compared to patients treated with the low-dose (6.7 months; hazard ratio $0.39 ; P=0.02)$. These data have been used to justify a Phase III clinical trial ${ }^{142}$.

Coxsackievirus. Coxsackievirus is a non-enveloped single-stranded RNA enterovirus that is a member of the Picornaviridae family. Coxsackievirus replicates in the cytosol without a DNA phase, eliminating the possibility of insertional mutagenesis during infection. There are two coxsackievirus subgroups, A and B, which are differentiated based on their pathogenesis in murine models. Coxsackievirus is further distinguished by over 23 serotypes in group A, with six distinct serotypes in group B. Coxsackievirus infections are generally asymptomatic, but sometimes manifest with common coldlike symptoms. Coxsackievirus utilizes ICAM-1 and DAF for cell entry ${ }^{75}$. Coxsackievirus A21 (Cavatak; Viralytics Ltd) has a natural tropism for cancer cells because some cancer cells, such as multiple myeloma, melanoma, and breast cancer cells, overexpress ICAM-1 and/or $\mathrm{DAF}^{45}$.

In addition to direct lysis of tumour cells, coxsackievirus has been shown to enhance the immune response, in part by promoting the release of DAMPs (for example, HMGB-1, calreticulin, and ATP ${ }^{143}$. Coxsackievirus infection promotes the infiltration of immune effector cells, including NK cells and $\mathrm{CD} 8^{+} \mathrm{T}$ cells, and enhances antigen presentation by activating dendritic cells ${ }^{143}$. Furthermore, it can increase the release of type I IFNs, which may enhance an antitumour immune response.

Key advantages of coxsackievirus as an oncolytic agent include the fact that the virus does not require complex genetic manipulation for safety or oncolytic activity, and that strong immune responses are induced with infection. A potential obstacle to the use of coxsackievirus as an oncolytic virus is that some people may have previously been exposed to native coxsackievirus, and may be immune to infection. However, antibodies against the different serotypes do not appear to be crossreactive; therefore, alternating treatment with different serotypes may prevent premature viral clearance. Clinical trials have been initiated with promising early results, suggesting therapeutic activity in melanoma with an acceptable safety profile ${ }^{144}$ (see Supplementary information S1 (table)). Combination studies of Cavatak and $\mathrm{T}$ cell checkpoint inhibitors in melanoma are anticipated.

Newcastle disease virus. NDV is a single-stranded RNA enveloped avian paramyxovirus that ranges in size from 100 to $500 \mathrm{~nm}$. NDV infects cells through plasma membrane fusion or through direct endocytosis of the virus. NDV replicates in the cytoplasm, so infected cells are not subject to insertional mutagenesis. The infection is asymptomatic in humans because the virus is highly sensitive to type I IFNs ${ }^{145}$, and NDV viral proteins trigger potent type I IFN responses ${ }^{146}$. As is the case with other oncolytic viruses, the sensitivity of NDV to type I IFNs confers cancer cell specificity ${ }^{145}$. Sensitivity to NDV-mediated apoptosis is also conferred by cancer cell overexpression of BCL- $\mathrm{X}_{\mathrm{L}}$ (REF. 52).

NDV induces cancer cell apoptosis and directly activates the innate immune system through increased cytokine production (type I IFN, RANTES (regulated on activation, normal $\mathrm{T}$ cell expressed and secreted; also known as CCL5), IL-12, and GM-CSF) and improved antigen presentation ${ }^{147}$. The NDV protein haemagglutininneuraminidase can act as a potent antigen that augments cytolytic T cell responses against infected tumour cells ${ }^{148}$. Thus, NDV-induced apoptosis of cancer cells results in the conversion of an immune-suppressive tumour microenvironment into a pro-inflammatory environment that supports antitumour immune responses. Interestingly, even low titres of NDV can limit tumour growth, suggesting that a key factor in its therapeutic activity may be the generation of an antitumour immune response ${ }^{149}$. Additionally, despite having a relatively small genome $(\sim 15 \mathrm{~kb})$, NDV does allow for the insertion of foreign genes. These can be inserted into non-coding regions without requiring the deletion of NDV viral genes, as demonstrated with GM-CSF expression ${ }^{150}$. Local delivery rather than systemic administration has been shown to enhance antitumour responses ${ }^{151}$.

Although numerous preclinical studies have suggested that NDV has antitumour activity against a wide variety of cancers, there are only a limited number of clinical trials in progress at present (TABLE 3; see Supplementary information S1 (table)). In contrast to many other oncolytic viruses, seropositivity against NDV is minimal in humans. However, the immune response generated against NDV can be potent, which has limited the maximum tolerated dose in humans ${ }^{152}$. Thus, dosing regimens that utilize an initial low dose followed by higher doses have been recommended for clinical trials. 
Reovirus. Reoviruses are double-stranded, non-enveloped RNA viruses with an outer surface icosahedral capsid and an inner core. Viral proliferation occurs in the cytoplasm of infected cells. In healthy cells, reovirus begins transcription by producing viral RNAs that aid in replication but also activate the PKR pathway ${ }^{57}$. However, in RAS-transformed cancer cells the PKR pathway is blocked. Thus, reovirus preferentially targets RAS-mutant cancers ${ }^{53}$. This natural tropism has led to many clinical trials against a multitude of cancer types, including gliomas, melanoma, ovarian cancer, and colorectal cancer (TABLE 3; see Supplementary information S1 (table)). The human population is commonly exposed to reovirus. Because most healthy cells are resistant to reoviral replication, pathology is typically subclinical. This does, however, complicate the use of reovirus as a potential oncolytic viral therapy. $70-100 \%$ of participants across several trials were shown to be seropositive for neutralizing antibodies against reovirus ${ }^{153,154}$.

Measles virus. Measles virus is a negative-stranded RNA paramyxovirus, measuring $150 \mathrm{~nm}$ in diameter, with a genome $(\sim 15 \mathrm{~kb})$ containing six genes that encode eight proteins. Measles virus uses the signalling lymphocytic activation molecule (SLAM) receptor, which is primarily expressed by lymphocytes, and/or CD46 to enter cells. Upon cell entry, measles virus remains within the cytoplasm where it undergoes replication, producing viral RNAs and capsid proteins. Measles virus propagates infection via cell-to-cell fusion, resulting in the formation of multicellular aggregates and ultimately cell death. However, measles virus causes serious illness in humans, thus requiring widespread vaccination to prevent disease. The pathology of the wild-type virus limits its use as an oncolytic therapeutic agent; however, attenuated strains are safer. Specifically, the Edmonston strain preferentially lyses cancer cells and enters through CD46 (REFS 41,42).

Case studies from the 1970s documented spontaneous tumour regression associated with coincident measles infection, especially in haematological malignancies ${ }^{155-157}$. More recently, the Edmonston strain of measles virus has been evaluated in several clinical trials for multiple tumour types, including GBM, multiple myeloma and ovarian cancer (TABLE 3; see Supplementary information S1 (table)). Similar to other oncolytic viruses, therapy with measles virus can be complicated by pre-existing neutralizing antibodies, as many patients may have been exposed to or vaccinated against measles virus. Vaccination has been associated with neutralizing antibodies and long-term memory responses, which can result in rapid clearance of the virus. Further clinical research is needed to better define the therapeutic activity of oncolytic measles viruses.

Poliovirus. Poliovirus is a non-enveloped, single-stranded RNA picornavirus measuring $30 \mathrm{~nm}$ in diameter. Poliovirus enters cells by binding to CD155, and following internalization the virus undergoes replication within the cytoplasm. Poliovirus is highly pathogenic in humans, resulting in paralytic poliomyelitis in $<1 \%$ of infected individuals owing to the destruction of anterior horn motor neurons following infection. Thus, poliovirus needs to be attenuated for use as an oncolytic vector. An attenuated strain (Sabin) has shown particular tropism for glioma cells, which is likely to be related to the upregulation of CD155 on these tumour cells ${ }^{158}$.

To reduce neurovirulence, poliovirus can be further attenuated by replacing the viral internal ribosome entry site (IRES) with an IRES from the related human rhino virus type 2 (HRV2); this construct has been termed PVS$\mathrm{RIPO}^{159}$. Additionally, replacing the poliovirus IRES with the HRV2 IRES enhances the selectivity of PVS-RIPO for GBM. HRV2 IRES binds to the DRBP76-NF45 heterodimer (comprising cellular double-stranded RNA-binding protein 76 (DRBP76; also known as ILF3) and nuclear factor of activated T cells $45 \mathrm{kDa}$ (NF45; also known as ILF2)). This interaction blocks viral replication in healthy neuronal cells, but does not occur in glioma cells ${ }^{160,161}$. The preclinical success of PVS-RIPO in GBM tumour models has led to ongoing Phase I clinical trials in GBM.

Other viruses. A large number of other oncolytic viruses have been proposed for cancer therapy. This includes VSV/rhabdovirus, Seneca Valley Virus ${ }^{32}$, parvovirus ${ }^{33}$, and retroviruses ${ }^{162}$. These agents are in early stages of clinical development and further clinical studies are needed to validate these approaches.

\section{Special issues in drug development}

Unique challenges to the development of oncolytic viruses as a new class of anticancer drugs include the need for more practical clinical trial designs and end point response assessment criteria, validated pharmacodynamic and pharmacokinetic (PK/PD) assays, biosafety issues and non-traditional regulatory, manufacturing and commercialization issues.

Pharmacodynamic considerations. Oncolytic viruses differ from standard drugs in several unique ways. They are live viruses and proliferate upon clinical administration; this can result in variable effective doses. At present, little data are available on correlating viral dose with in vivo replicative potential and therapeutic response. Further investigations relating to viral replication and clinical response in relevant preclinical models and in clinical trials will be important for establishing safe and effective dosing guidelines.

Oncolytic viruses are not removed as a result of cell metabolism or binding to circulating proteins; rather, they are subject to host antiviral immune responses. Multiple factors need to be considered in this regard, including pre-existing neutralizing antibody titres, virus-specific memory $\mathrm{T}$ cell responses, the potential for disabling some viruses by haemagglutinin binding and the innate ability of some virus to evade immune detection. In addition, many tumour microenvironments are highly immune-suppressive, which can limit the host immune response. Further studies to evaluate both preexisting antiviral immunity and the induction of posttreatment immune responses against the viral vector and tumour-associated antigens will be important to better understand the dynamics of how oncolytic viruses are cleared and controlled in patients with cancer. 
Biosafety considerations. Oncolytic viruses have thus far been associated with a relatively tolerable safety profile in numerous clinical trials conducted across a wide range of cancers. Nonetheless, the replicative potential of these agents requires attention to infection control issues, including procedures for the safe storage, preparation, handling and administration of the virus. The true potential for infection depends on the nature of the virus and co-morbid conditions within patients, close household contacts and health-care workers who may be exposed to the virus. In addition, many oncolytic viruses contain recombinant DNA elements, and so the potential impact of such gene segments and the potential for their recombination with wild-type viruses in the environment are theoretical concerns.

Although there are no universally accepted standards with respect to the biosafety of oncolytic viruses, the developmental plan should include policies for the safe handling and storage of the agent. Guidelines for health-care workers, especially pharmacists who prepare the agent and physicians or nurses who administer the agent, should be established. Most agents can be safely managed with standard universal precautions but additional educational materials need to be developed. For each virus, there should be clear instructions for how to deal with accidental spills, cleaning treatment rooms in between patient visits and instructions on possible antidotes in the event of an accidental exposure. In some cases, warnings should be issued for patients and health-care workers to avoid contact with the agent. For example, immunocompromised individuals and pregnant women should not be exposed to vaccinia virus. Plans for the management of the virus administration site should also be clearly delineated for patients and health-care workers. In the event of possible contamination of bandages and dressings, a plan for proper disposal in health-care settings and at home should be developed.

As oncolytic viruses are new agents with potential for dissemination, many plans may need to consider expanded surveillance programmes. At present it is not clear how regulatory agencies will manage the biosafety issues, but consideration of post-marketing risk evaluation and mitigation strategy (REMS) processes may be indicated. Despite the theoretical concerns, it should be noted that there have been no reports of household transmission of oncolytic viruses in the literature to date. It is also important to note that many oncolytic viruses are attenuated and the native strains are often ubiquitous in the environment. There have, however, been isolated cases of exposure of health-care professionals while handling oncolytic viruses in the laboratory or before patient administration. These cases have generally been few and without significant sequelae, although some individuals have required treatment with antiviral agents.

Clinical trial design and response assessment. Recent evidence has suggested that the kinetics of immunemediated responses may be much slower when compared to therapeutic agents that directly kill tumour cells. Activation of the immune system results in an indirect method of tumour cell killing, which requires the priming and expansion of immune effector cells, most notably
$\mathrm{CD} 8^{+}$cytotoxic $\mathrm{T}$ cells, and time for such cells to undergo homeostatic expansion, trafficking to sites of tumour growth, lytic destruction of cancer cells and inflammatory clearance of the necrotic tumour (FIG. 2). This may result in what has been termed 'pseudo-progression' and clinical trials must consider non-traditional end points that can account for the emergence of delayed therapeutic responses ${ }^{163}$. Pseudo-progression may complicate the interpretation of clinical results and should be considered in future oncolytic virus trials.

In addition, oncolytic viruses represent a challenge with respect to subject eligibility, dosing and biodistribution assessment when designing clinical trials. As many clinical studies have utilized local delivery of the virus to patients, tumours must be accessible for injection. To date, virus dosing has typically been based on the maximum concentration of virus possible, with current purification techniques and the volume of tumour as determined before each virus administration. This can be quite cumbersome and few studies have adequately explored the association between virus dose, therapeutic response and occurrences of adverse events ${ }^{142,164,165}$. Nonetheless, because oncolytic viruses can replicate within tumour tissues, even small doses may result in significant clinical activity provided the virus is not rapidly cleared by the immune system. This was observed in clinical trials with oncolytic poliovirus and T-VEC ${ }^{3}$. Finally, individual assays to assess virus distribution are needed to evaluate the pharmacodynamics of virus delivery. Ideally, this should include data on the presence of the virus within targeted cells (for example, cancer cells), lack of viral replication in non-target cells, virus shedding within body fluids, evidence of latent infection, viral clearance, and virus-specific and tumour-specific humoral and cellular immune responses. Although many trials have made progress in establishing such assays, there is no standard agreement within the field on how to validate these correlative end points.

Several strategies for clinical assessment of oncolytic viruses in vivo have been developed. The most common methods for determining tissue uptake have used the expression of fluorescent dyes, such as green fluorescent protein (GFP) or luciferase, in the viral vector to quantitate viral transcription within infected cells ${ }^{166}$. This technique, however, is challenging in patients as it requires the inclusion of a foreign transgene with unknown clinical consequences, potential for immune recognition and difficulty in imaging fluorescent activity from deep tissue layers. By contrast, measles virus and HSV-1 have been engineered to express other proteins or enzymes that do not rely on fluorescence for in vivo monitoring. Measles virus has been engineered to encode the soluble extracellular domain of human CEA upstream of the nucleoprotein gene. This allows quantitative monitoring of viral gene expression through serial peripheral blood measurement of CEA levels ${ }^{167}$. Another interesting strategy utilized a measles virus expressing human thyroidal sodium iodide symporter (NIS) ${ }^{168}$. The NIS concentrates radioactive iodine, which can be monitored using single photon emission computed tomography (SPECT) or positron emission tomography (PET) imaging. An HSV-1 encoding viral TK and a mutated dopamine $\mathrm{D}_{2}$ receptor 
was used to trap radioactive tracers for monitoring virus location and replication ability ${ }^{169}$. In this system, viral TK phosphorylates injected radioactive ${ }^{131}$ I-labelled 2 '-fluo ro-2'-deoxy-1-beta-D-arabinofuranosyl-5-iodo-uracil (FIAU), which traps the radioactive signal within the cell ${ }^{170}$. The mutated dopamine $\mathrm{D}_{2}$ receptor acts in a similar fashion by trapping a radioactive tracer that can be monitored using PET imaging. In addition to imaging, tumour biopsy procedures can be performed, when accessible and appropriate, to determine viral titres in target and nontarget tissues. Tumour biopsy samples can provide both functional data as well as information about the kinetics and propagation of the oncolytic virus. Collection of other body tissues, including blood, saliva and urine, may also provide indicators of viral replication and will be critical to ensure safety by determining the bioshedding dynamics of therapeutic oncolytic viruses.

Regulatory and commercialization issues. The live replicating nature of oncolytic viruses poses unique regulatory and manufacturing issues. Most viruses are propagated in tissue cultures and this requires methods for high-titre virus production, testing for adventitial pathogens, and assessment of virus purity and replication potential. Thus, procedures for laboratory safety during manufacturing and vialing, product validation and purity, and quality of design that results from generating biologics in cell culture must be considered. For some viruses it has been difficult to generate very high titre lysates required for clinical dosing, and this can be a challenge for biotechnology manufacturing. These aspects have been reviewed extensively elsewhere ${ }^{171-173}$. Viral vaccines have provided the templates for the manufacture and regulation of oncolytic viruses. The FDA has published a draft guidance document titled "Guidance for Industry: Design and Analysis of Shedding Studies for Virus or Bacteria-Based Gene Therapy and Oncolytic Products". Other relevant documents include the "European Directorate for the Quality of Medicines including Cell substrates for the production of vaccines for human use" (REF. 174) and "Tests for extraneous agents in viral vaccines for human use" (REF. 175). These provide guidelines for quality control testing of all materials and cells used in the generation of oncolytic viruses. Furthermore, intermediates and viral batches need to be tested for contamination and efficacy. However, removing contaminants, especially microbial or viral contaminants, proves to be more difficult for oncolytic viruses than for recombinant proteins because the methods used to remove these contaminants may also target the oncolytic virus. The quality of oncolytic virus preparation can be tested in control cells with a standard readout, in combination with a virus-specific neutralizing antibody to confirm that the effect is mediated by the virus. This approach has been adapted from the methods used to test human viral vaccines. This infrastructure should provide the additional framework needed for the widespread commercialization of oncolytic viruses for cancer therapy.

\section{Future directions}

The demonstration of a significant improvement in durable responses for patients with melanoma who were treated with T-VEC has revolutionized the field of anticancer therapy with oncolytic viruses. However, the development of oncolytic viruses as therapeutic agents requires careful attention to establish appropriate clinical trial designs, dosing regimens, pharmacodynamic assays, educational programmes addressing biosafety concerns, as well as new manufacturing and regulatory pathways. Likewise, careful attention to patient selection will also be an important consideration. For example, immunocompromised patients may not be good candidates because oncolytic virus-mediated anti-tumour immunity could be compromised in these patients.

Nevertheless, oncolytic viruses have been associated with a very favourable risk-benefit ratio, and continued development of this new class of drugs can be anticipated, with particular interest in combination approaches. Oncolytic virus immunotherapy is a highly promising approach and introduces a new class of drugs for treating patients with cancer.
1. Moore, A. E. The destructive effect of the virus of Russian Far East encephalitis on the transplantable mouse sarcoma 180. Cancer 2, 525-534 (1949).

2. Moore, A. E. Effect of inoculation of the viruses of influenza $A$ and herpes simplex on the growth of transplantable tumors in mice. Cancer 2, 516-524 (1949).

3. Andtbacka, R. H. et al. Talimogene laherparepvec improves durable response rate in patients with advanced melanoma. J. Clin. Oncol. http://dx.doi.org/10.1200/JCO.2014.58.3377 (2015) Phase III clinical trial that led to the pending FDA approval of T-VEC for clinical use in the United States for melanoma.

4. Anthony, S. J. et al. A strategy to estimate unknown viral diversity in mammals. mBio 4, e00598-e00513 (2013).

5. Hanahan, D. \& Weinberg, R. A. Hallmarks of cancer: the next generation. Cell 144, 646-674 (2011).

6. Martuza, R. L., Malick, A., Markert, J. M., Ruffner, K. L. \& Coen, D. M. Experimental therapy of human glioma by means of a genetically engineered virus mutant. Science 252, 854-856 (1991).

First paper showing that viruses can be engineered to improve oncolytic activity.

7. Garber, K. China approves world's first oncolytic virus therapy for cancer treatment. J. Natl Cancer Inst. 98 , 298-300 (2006)
$\mathrm{H} 101$ is the first oncolytic virus approved for clinical use in China.

8. Alvarez-Breckenridge, C., Kaur, B. \& Chiocca, E. A. Pharmacologic and chemical adjuvants in tumor virotherapy. Chem. Rev. 109, 3125-3140 (2009).

9. Uchida, H. et al. Effective treatment of an orthotopic xenograft model of human glioblastoma using an EGFR-retargeted oncolytic herpes simplex virus. Mol. Ther. 21, 561-569 (2013).

10. Meurs, E. et al. Molecular cloning and characterization of the human double-stranded RNA-activated protein kinase induced by interferon. Cell 62, 379-390 (1990)

11. Elde, N. C., Child, S. J., Geballe, A. P. \& Malik, H. S. Protein kinase $\mathrm{R}$ reveals an evolutionary model for defeating viral mimicry. Nature 457, 485-489 (2009). Describes how cellular PKR has a major role in host cell responses to virus infection.

12. Clemens, M. J. Targets and mechanisms for the regulation of translation in malignant transformation. Oncogene 23, 3180-3188 (2004).

13. Zamarin, D. et al. Localized oncolytic virotherapy overcomes systemic tumor resistance to immune checkpoint blockade immunotherapy. Sci. Transl Med. 6, 226ra32 (2014).

NDV requires both CD8 T cells and NK cells for systemic anti-tumour effects.
14. Karre, K., Ljunggren, H. G., Piontek, G. \& Kiessling, R. Selective rejection of $\mathrm{H}$-2-deficient lymphoma variants suggests alternative immune defence strategy. Nature 319, 675-678 (1986).

15. Ljunggren, H. G. \& Karre, K. Host resistance directed selectively against $\mathrm{H}$-2-deficient lymphoma variants. Analysis of the mechanism. J. Exp. Med. 162 1745-1759 (1985).

16. Ikeda, K. et al. Oncolytic virus therapy of multiple tumors in the brain requires suppression of innate and elicited antiviral responses. Nat. Med. 5, 881-887 (1999). Antibodies and the innate immune system limit persistence and activity of oncolytic viruses.

17. Wakimoto, H. et al. The complement response against an oncolytic virus is species-specific in its activation pathways. Mol. Ther. 5, 275-282 (2002).

18. Alvarez-Breckenridge, C. A. et al. NK cells impede glioblastoma virotherapy through NKp30 and NKp46 natural cytotoxicity receptors. Nat. Med. 18, 1827-1834 (2012).

19. Ruffell, B., Affara, N. I. \& Coussens, L. M. Differential macrophage programming in the tumor microenvironment. Trends Immunol. 33, 119-126 (2012).

20. Talmadge, J. E. \& Gabrilovich, D. I. History of myeloid-derived suppressor cells. Nat. Rev. Cancer 13, 739-752 (2013). 
21. Gajewski, T. F., Schreiber, H. \& Fu, Y. X. Innate and adaptive immune cells in the tumor microenvironment. Nat. Immunol. 14, 1014-1022 (2013).

22. Di Paolo, N. C. et al. Virus binding to a plasma membrane receptor triggers interleukin- $1 \alpha$-mediated proinflammatory macrophage response in vivo. Immunity 31, 110-121 (2009).

23. Prestwich, R. J. et al. The case of oncolytic viruses versus the immune system: waiting on the judgment of Solomon. Hum. Gene Ther. 20, 1119-1132 (2009).

24. Bridle, B. W. et al. Potentiating cancer immunotherapy using an oncolytic virus. Mol. Ther. 18, 1430-1439 (2010).

25. Kanerva, A. et al. Antiviral and antitumor T-cell immunity in patients treated with GM-CSF-coding oncolytic adenovirus. Clin. Cancer Res. 19 2734-2744 (2013).

Analysis of T cell responses from patients treated with an oncolytic adenovirus suggests that these viruses cause epitope spreading.

26. Carlsten, M. et al. Primary human tumor cells expressing CD 155 impair tumor targeting by downregulating DNAM-1 on NK cells. J. Immunol. 183 4921-4930 (2009).

27. Snyder, A. et al. Genetic basis for clinical response to CTLA-4 blockade in melanoma. N. Engl. J. Med. 371 2189-2199 (2014)

Describes a relationship between tumour cell mutation load, emergence of neo-antigens and clinical response to T cell checkpoint inhibitors.

28. Schietinger, A., Philip, M., Liu, R. B., Schreiber, K. \& Schreiber, $H$. Bystander killing of cancer requires the cooperation of $\mathrm{CD}^{+}$and $\mathrm{CD} 8^{+} \mathrm{T}$ cells during the effector phase. J. Exp. Med. 207, 2469-2477 (2010).

29. Nguyen, A., Ho, L. \& Wan, Y. Chemotherapy and oncolytic virotherapy: advanced tactics in the war against cancer. Front. Oncol. 4, 145 (2014).

30. Mok, W., Boucher, Y. \& Jain, R. K. Matrix metalloproteinases-1 and - 8 improve the distribution and efficacy of an oncolytic virus. Cancer Res. 67 10664-10668 (2007)

31. Shen, B. H. \& Hermiston, T. W. Effect of hypoxia on Ad5 infection, transgene expression and replication. Gene Ther. 12, 902-910 (2005)

32. Rudin, C. M. et al. Phase I clinical study of Seneca Valley Virus (SVV-001), a replication-competent picornavirus, in advanced solid tumors with neuroendocrine features. Clin. Cancer Res. 17 888-895 (2011)

33. Geletneky, K. et al. Phase I/lla study of intratumoral/ intracerebral or intravenous/intracerebral administration of Parvovirus H-1 (ParvOryx) in patients with progressive primary or recurrent glioblastoma multiforme: ParvOryx01 protocol. BMC Cancer 12, 99 (2012)

34. Chiocca, E. A. et al. A phase I open-label, dose escalation, multi-institutional trial of injection with an E1B-attenuated adenovirus, ONYX-015, into the peritumoral region of recurrent malignant gliomas, in the adjuvant setting. Mol. Ther. 10, 958-966 (2004)

35. Eck, S. L. et al. Treatment of advanced CNS malignancies with the recombinant adenovirus H5.010RSVTK a phase I trial. Hum Gene Ther 7, 1465-1482 (1996).

36. Fueyo, J. et al. A mutant oncolytic adenovirus targeting the $\mathrm{Rb}$ pathway produces anti-glioma effect in vivo. Oncogene 19, 2-12 (2000).

37. Heise, C. et al. An adenovirus E1 A mutant that demonstrates potent and selective systemic antitumoral efficacy. Nat. Med. 6, 1134-1139 (2000).

38. Lopez, M. V. et al. Tumor associated stromal cells play a critical role on the outcome of the oncolytic efficacy of conditionally replicative adenoviruses. PLOS ONE 4 e5119 (2009).

39. Zamarin, D. \& Palese, P. Oncolytic Newcastle disease virus for cancer therapy: old challenges and new directions. Future Microbiol. 7, 347-367 (2012)

40. Yu, Z. et al. Enhanced nectin-1 expression and herpes oncolytic sensitivity in highly migratory and invasive carcinoma. Clin. Cancer Res. 11, 4889-4897 (2005).

41. Dorig, R. E., Marcil, A., Chopra, A. \& Richardson, C. D The human CD46 molecule is a receptor for measles virus (Edmonston strain). Cell 75, 295-305 (1993).

42. Anderson, B. D., Nakamura, T., Russell, S. J. \& Peng, K. W. High CD46 receptor density determines preferential killing of tumor cells by oncolytic measles virus. Cancer Res. 64, 4919-4926 (2004).

43. Guo, P. et al. ICAM-1 as a molecular target for triple negative breast cancer. Proc. Natl Acad. Sci. USA 111 14710-14715 (2014).
44. Au, G. G., Lincz, L. F., Enno, A. \& Shafren, D. R. Oncolytic Coxsackievirus A21 as a novel therapy for multiple myeloma. Br. J. Haematol. 137, 133-141 (2007).

45. Shafren, D. R. et al. Systemic therapy of malignant human melanoma tumors by a common cold-producing enterovirus, coxsackievirus a2 1 . Clin. Cancer Res. 10, 53-60 (2004).

46. Shafren, D. R., Sylvester, D., Johansson, E. S., Campbell, I. G. \& Barry, R. D. Oncolysis of human ovarian cancers by echovirus type 1 . Int. J. Cancer 115, 320-328 (2005)

47. Rea, V. E. et al. $67 \mathrm{kDa}$ laminin receptor: structure function and role in cancer and infection. Infez Med. 20 (Suppl. 2), 8-12 (2012).

48. You, Z. et al. Coxsackievirus-adenovirus receptor expression in ovarian cancer cell lines is associated with increased adenovirus transduction efficiency and transgene expression. Cancer Gene Ther. 8, 168-175 (2001).

49. Liapis, H., Adler, L. M., Wick, M. R. \& Rader, J. S Expression of $\alpha_{v} \beta 3$ integrin is less frequent in ovarian epithelial tumors of low malignant potential in contrast to ovarian carcinomas. Hum. Pathol. 28 , 443-449 (1997).

50. Morizono, K. et al. Lentiviral vector retargeting to P-glycoprotein on metastatic melanoma through intravenous injection. Nat. Med. 11, 346-352 (2005)

51. Hammond, A. L. et al. Single-chain antibody displayed on a recombinant measles virus confers entry through the tumor-associated carcinoembryonic antigen J. Virol. 75, 2087-2096 (2001)

52. Mansour, M., Palese, P. \& Zamarin, D. Oncolytic specificity of Newcastle disease virus is mediated by selectivity for apoptosis-resistant cells. J. Virol. 85 6015-6023 (2011)

53. Strong, J. E., Coffey, M. C., Tang, D., Sabinin, P. \& Lee, P. W. The molecular basis of viral oncolysis: usurpation of the Ras signaling pathway by reovirus. EMBO J. 17, 3351-3362 (1998).

54. Parato, K. A. et al. The oncolytic poxvirus JX-594 selectively replicates in and destroys cancer cells driven by genetic pathways commonly activated in cancers. Mol. Ther. 20, 749-758 (2012).

The oncolytic vaccinia virus, JX-594, showed enhanced tumour selectivity based on aberrant EGFR-RAS signalling in cancer cells.

55. Farassati, F., Yang, A. D. \& Lee, P. W. Oncogenes in Ras signalling pathway dictate host-cell permissiveness to herpes simplex virus 1. Nat. Cell Biol. 3, 745-750 (2001).

56. Cuddington, B. P. \& Mossman, K. L. Permissiveness of human cancer cells to oncolytic bovine herpesvirus 1 is mediated in part by KRAS activity. J. Virol. $\mathbf{8 8}$, 6885-6895 (2014).

57. Bischoff, J. R. \& Samuel, C. E. Mechanism of interferon action. Activation of the human P1/elF- $2 \alpha$ protein kinase by individual reovirus s-class mRNAs: s1 mRNA is a potent activator relative to $\$ 4$ mRNA. Virology 172, 106-115 (1989)

58. Wilden, H., Fournier, P., Zawatzky, R. \& Schirrmacher, V. Expression of RIG-I, IRF3, IFN- $\beta$ and IRF7 determines resistance or susceptibility of cells to infection by Newcastle Disease Virus. Int. J. Oncol. 34 971-982 (2009).

59. Bell, J. C., Lichty, B. \& Stojdl, D. Getting oncolytic virus therapies off the ground. Cancer Cell 4, 7-11 (2003)

60. Vaha-Koskela, M. J., Heikkila, J. E. \& Hinkkanen, A. E. Oncolytic viruses in cancer therapy. Cancer Lett. 254 , 178-216 (2007)

61. Stojdl, D. F. et al. Exploiting tumor-specific defects in the interferon pathway with a previously unknown oncolytic virus. Nat. Med. 6, 821-825 (2000).

62. Takaoka, A. et al. Integration of interferon- $\alpha / \beta$ signalling to $\mathrm{p} 53$ responses in tumour suppression and antiviral defence. Nature 424, 516-523 (2003)

63. DeWeese, T. L. et al. A phase I trial of CV706, a replication-competent, PSA selective oncolytic adenovirus, for the treatment of locally recurrent prostate cancer following radiation therapy. Cancer Res. 61, 7464-7472 (2001)

64. Chang, J. et al. A Phase I study of KH901, a conditionally replicating granulocyte-macrophage colony-stimulating factor: armed oncolytic adenovirus for the treatment of head and neck cancers. Cancer Biol. Ther. 8, 676-682 (2009).

65. Ramesh, N. et al. CG0070, a conditionally replicating granulocyte macrophage colony-stimulating factor armed oncolytic adenovirus for the treatment of bladder cancer. Clin. Cancer Res. 12, 305-313 (2006).
66. Neuman, E., Flemington, E. K., Sellers, W. R. \& Kaelin, W. G. Jr. Transcription of the E2F-1 gene is rendered cell cycle dependent by E2F DNA-binding sites within its promoter. Mol. Cell. Biol. 14 6607-6615 (1994)

67. Post, D. E. et al. Targeted cancer gene therapy using a hypoxia inducible factor dependent oncolytic adenovirus armed with interleukin-4. Cancer Res 67, 6872-6881 (2007)

68. Leber, M. F et al. MicroRNA-sensitive oncolytic measles viruses for cancer-specific vector tropism. Mol. Ther. 19, 1097-1106 (2011)

69. Freeman, A. I. et al. Phase I/II trial of intravenous NDV-HUJ oncolytic virus in recurrent glioblastoma multiforme. Mol. Ther. 13, 221-228 (2006).

70. Brown, S. M., MacLean, A. R., McKie, E. A. \& Harland, J. The herpes simplex virus virulence factor ICP34.5 and the cellular protein MyD116 complex with proliferating cell nuclear antigen through the 63-amino-acid domain conserved in ICP34.5, MyD116, and GADD34. J. Virol. 71, 9442-9449 (1997).

71. Harland, J., Dunn, P., Cameron, E., Conner, J. \& Brown, S. M. The herpes simplex virus (HSV) protein ICP34.5 is a virion component that forms a DNAbinding complex with proliferating cell nuclear antigen and HSV replication proteins. J. Neuroviro 9, 477-488 (2003).

72. Orvedahl, A. et al. HSV-1 ICP34.5 confers neurovirulence by targeting the Beclin 1 autophagy protein. Cell Host Microbe 1, 23-35 (2007).

73. Davis, K. L., Korom, M. \& Morrison, L. A. Herpes simplex virus 2 ICP34.5 confers neurovirulence by regulating the type I interferon response. Virology 468-470, 330-339 (2014).

74. Liu, B. L. et al. ICP34.5 deleted herpes simplex virus with enhanced oncolytic, immune stimulating, and anti-tumour properties. Gene Ther. 10, 292-303 (2003).

75. Shafren, D. R., Dorahy, D. J., Ingham, R. A Burns, G. F. \& Barry, R. D. Coxsackievirus A21 binds to decay-accelerating factor but requires intercellular adhesion molecule 1 for cell entry. J. Virol. 71, 4736-4743 (1997).

76. Colamonici, O. R., Domanski, P., Sweitzer, S. M., Larner, A. \& Buller, R. M. Vaccinia virus B18R gene encodes a type I interferon-binding protein that blocks interferon $\alpha$ transmembrane signaling. J. Biol. Chem. 270, 15974-15978 (1995)

77. Gulley, J. L. et al. Pilot study of vaccination with recombinant CEA-MUC-1-TRICOM poxviral-based vaccines in patients with metastatic carcinoma. Clin. Cancer Res. 14, 3060-3069 (2008)

78. Kirn, D. H., Wang, Y., Le Boeuf, F., Bell, J. $\Sigma$ Thorne, S. H. Targeting of interferon- $\beta$ to produce a specific, multi-mechanistic oncolytic vaccinia virus. PLoS Med. 4, e353 (2007).

79. Toda, M., Martuza, R. L. \& Rabkin, S. D. Tumor growth inhibition by intratumoral inoculation of defective herpes simplex virus vectors expressing granulocytemacrophage colony-stimulating factor. Mol. Ther. $\mathbf{2}$ 324-329 (2000)

80. Hu, J. C. et al. A phase I study of OncoVEXGM-CSF, a second-generation oncolytic herpes simplex virus expressing granulocyte macrophage colonystimulating factor. Clin. Cancer Res. 12, 6737-6747 (2006).

81. Burke, J. M. et al. A first in human phase 1 study of CG0070, a GM-CSF expressing oncolytic adenovirus, for the treatment of nonmuscle invasive bladder cancer. J. Urol. 188, 2391-2397 (2012).

82. Mastrangelo, M. J. et al. Intratumoral recombinant GM-CSF-encoding virus as gene therapy in patients with cutaneous melanoma. Cancer Gene Ther. $\mathbf{6}$, 409-422 (1999)

83. Li, J. L. et al. A phase I trial of intratumoral administration of recombinant oncolytic adenovirus overexpressing HSP70 in advanced solid tumor patients. Gene Ther. 16, 376-382 (2009).

84. Tomazin, R. et al. Herpes simplex virus type 2 ICP47 inhibits human TAP but not mouse TAP. J. Virol. 72 2560-2563 (1998).

85. Heise, C. et al. ONYX-015, an E1B gene-attenuated adenovirus, causes tumor-specific cytolysis and antitumoral efficacy that can be augmented by standard chemotherapeutic agents. Nat. Med. 3 639-645 (1997)

86. Yuan, Z. Y., Zhang, L., Li, S., Qian, X. Z. \& Guan, Z. Z Safety of an E1B deleted adenovirus administered intratumorally to patients with cancer. Ai Zheng 22, 310-313 (in Chinese) (2003). 
87. Poppers, J., Mulvey, M., Khoo, D. \& Mohr, I. Inhibition of PKR activation by the proline-rich RNA binding domain of the herpes simplex virus type 1 Us 11 protein. J. Virol. 74, 11215-11221 (2000).

88. Di Piazza, M. et al. Cytosolic activation of cathepsins mediates parvovirus $\mathrm{H}-1$-induced killing of cisplatin and TRAIL-resistant glioma cells. J. Virol. $\mathbf{8 1}$ 4186-4198 (2007).

89. Grekova, S. et al. Immune cells participate in the oncosuppressive activity of parvovirus $\mathrm{H}-1 \mathrm{PV}$ and are activated as a result of their abortive infection with this agent. Cancer Biol. Ther. 10, 1280-1289 (2010)

90. Bar, S., Daeffler, L., Rommelaere, J. \& Nuesch, J. P. Vesicular egress of non-enveloped lytic parvoviruses depends on gelsolin functioning. PLoS Pathog. 4 e 1000126 (2008)

91. Sova, P. et al. A tumor-targeted and conditionally replicating oncolytic adenovirus vector expressing TRAIL for treatment of liver metastases. Mol. Ther 9, 496-509 (2004).

92. Hirvinen, M. et al. Immunological effects of a tumor necrosis factor $\alpha$-armed oncolytic adenovirus Hum. Gene Ther. 26, 134-144 (2015).

93. Freytag, S. O., Rogulski, K. R., Paielli, D. L., Gilbert, J. D. \& Kim, J. H. A novel three-pronged approach to kill cancer cells selectively: concomitant viral, double suicide gene, and radiotherapy. Hum. Gene Ther. 9, 1323-1333 (1998)

94. Freytag, S. O. et al. Phase I study of replicationcompetent adenovirus-mediated double suicide gene therapy for the treatment of locally recurrent prostate cancer. Cancer Res. 62, 4968-4976 (2002)

95. Foloppe, J. et al. Targeted delivery of a suicide gene to human colorectal tumors by a conditionally replicating vaccinia virus. Gene Ther 15 1361-1371 (2008)

96. Kubo, H. et al. Phase I dose escalation clinical trial of adenovirus vector carrying osteocalcin promoterdriven herpes simplex virus thymidine kinase in localized and metastatic hormone-refractory prostate cancer. Hum. Gene Ther. 14, 227-241 (2003).

97. Alvarez, R. D. \& Curiel, D. T. A phase I study of recombinant adenovirus vector-mediated intraperitoneal delivery of herpes simplex virus thymidine kinase (HSV-TK) gene and intravenous ganciclovir for previously treated ovarian and extraovarian cancer patients. Hum. Gene Ther. 8, 597-613 (1997).

98. Aghi, M., Chou, T. C., Suling, K., Breakefield, X. O. \& Chiocca, E. A. Multimodal cancer treatment mediated by a replicating oncolytic virus that delivers the oxazaphosphorine/rat cytochrome P450 2B1 and ganciclovir/herpes simplex virus thymidine kinase gene therapies. Cancer Res. 59, 3861-3865 (1999).

99. Boviatsis, E. J. et al. Long-term survival of rats harboring brain neoplasms treated with ganciclovir and a herpes simplex virus vector that retains an intact thymidine kinase gene. Cancer Res. 54, 5745-5751 (1994).

100. Freytag, S. O. et al. Phase I study of replicationcompetent adenovirus-mediated double-suicide gene therapy in combination with conventional-dose threedimensional conformal radiation therapy for the treatment of newly diagnosed, intermediate- to highrisk prostate cancer. Cancer Res. 63, 7497-7506 (2003).

101. Doronin, K. et al. Tumor-specific, replicationcompetent adenovirus vectors overexpressing the adenovirus death protein. J. Virol. 74, 6147-6155 (2000).

102. Cattaneo, R., Miest, T., Shashkova, E. V. \& Barry, M. A Reprogrammed viruses as cancer therapeutics: targeted, armed and shielded. Nat. Rev. Microbiol. 6, 529-540 (2008)

103. Tesfay, M. Z. et al. PEGylation of vesicular stomatitis virus extends virus persistence in blood circulation of passively immunized mice. J. Virol. 87, 3752-3759 (2013).

104. O'Riordan, C. R. et al. PEGylation of adenovirus with retention of infectivity and protection from neutralizing antibody in vitro and in vivo. Hum. Gene Ther. 10, 1349-1358 (1999).

105. Morrison, J. et al. Virotherapy of ovarian cancer with polymer-cloaked adenovirus retargeted to the epidermal growth factor receptor. Mol. Ther. 16, 244-251 (2008)

106. Mader, E. K. et al. Mesenchymal stem cell carriers protect oncolytic measles viruses from antibody neutralization in an orthotopic ovarian cancer therapy model. Clin. Cancer Res. 15, 7246-7255 (2009).
107. Willmon, C et al. Cell carriers for oncolytic viruses: Fed Ex for cancer therapy. Mol. Ther. 17, 1667-1676 (2009)

108. Berger, C. et al. Expression of herpes simplex virus ICP47 and human cytomegalovirus US11 prevents recognition of transgene products by $\mathrm{CD} 8$ cytotoxic T lymphocytes. J. Virol. 74, 4465-4473 (2000)

109. Fulci, G. et al. Cyclophosphamide enhances glioma virotherapy by inhibiting innate immune responses. Proc. Natl Acad. Sci. USA 103, 12873-12878 (2006)

110. Bilbao, R. et al. A blood-tumor barrier limits gene transfer to experimental liver cancer: the effect of vasoactive compounds. Gene Ther. 7, 1824-1832 (2000).

111. Eisenberg, D. P. et al. Hyperthermia potentiates oncolytic herpes viral killing of pancreatic cance through a heat shock protein pathway. Surgery 148, 325-334 (2010).

112. Ingemarsdotter, C. K. et al. Low-dose paclitaxel synergizes with oncolytic adenoviruses via mitotic slippage and apoptosis in ovarian cancer. Oncogene 29, 6051-6063 (2010)

113. Deguchi, T et al Combination of the tumer angiogenesis inhibitor bevacizumab and intratumoral oncolytic herpes virus injections as a treatment strategy for human gastric cancers. Hepatogastroenterology 59, 1844-1850 (2012).

114. McKee, T. D. et al. Degradation of fibrillar collagen in a human melanoma xenograft improves the efficacy of an oncolytic herpes simplex virus vector. Cancer Res. 66, 2509-2513 (2006)

115. Ganesh, S., Gonzalez-Edick, M., Gibbons, D., Van Roey, M. \& Jooss, K. Intratumoral coadministration of hyaluronidase enzyme and oncolytic adenoviruses enhances virus potency in metastatic tumor models. Clin. Cancer Res. 14, 3933-3941 (2008). Oncolytic viruses have the potential to be engineered to overcome the physical barriers within the tumour microenvironment.

116. Guedan, S. et al. Hyaluronidase expression by an oncolytic adenovirus enhances its intratumoral spread and suppresses tumor growth. Mol. Ther 18, 1275-1283 (2010)

117. Ebert, O. et al. Syncytia induction enhances the oncolytic potential of vesicular stomatitis virus in virotherapy for cancer. Cancer Res. 64, 3265-3270 (2004).

118. Zhang, J., Frolov, I. \& Russell, S. J. Gene therapy for malignant glioma using Sindbis vectors expressing a fusogenic membrane glycoprotein. J. Gene Med. $\mathbf{6}$, 1082-1091 (2004).

119. Springfeld, C. et al. Oncolytic efficacy and enhanced safety of measles virus activated by tumor-secreted matrix metalloproteinases. Cancer Res. 66 7694-7700 (2006)

120. He, B., Gross, M. \& Roizman, B. The $\gamma, 34.5$ protein of herpes simplex virus 1 complexes with protein phosphatase $1 \alpha$ to dephosphorylate the $\alpha$ subunit of the eukaryotic translation initiation factor 2 and preclude the shutoff of protein synthesis by doublestranded RNA-activated protein kinase. Proc. Natl Acad. Sci. USA 94, 843-848 (1997).

121. Kaufman, H. L. et al. Local and distant immunity induced by intralesional vaccination with an oncolytic herpes virus encoding GM-CSF in patients with stage IIIc and IV melanoma. Ann. Surg. Oncol. 17, 718-730 (2010).

122. Kaufman, H. L. \& Bines, S. D. OPTIM trial: a Phase III trial of an oncolytic herpes virus encoding GM-CSF for unresectable stage III or IV melanoma. Future Oncol. 6, 941-949 (2010)

123. Puzanov, I. et al. Survival, safety, and response patterns in a phase $1 \mathrm{~b}$ multicenter trial of talimogene laherparepvec (T-VEC) and ipilimumab (ipi) in previously untreated, unresected stage IIIB-IV melanoma. J. Clin. Oncol. 33, S9063 (2015).

124. Bischoff, J. R. et al. An adenovirus mutant that replicates selectively in p53-deficient human tumor cells. Science 274, 373-376 (1996) Describes the ability of adenovirus to selectively replicate in p53-deficient tumour cells.

125. Rao, L. et al. The adenovirus E1A proteins induce apoptosis, which is inhibited by the E1B 19-kDa and $\mathrm{Bcl}-2$ proteins. Proc. Natl Acad. Sci. USA 89 , 7742-7746 (1992)

126. Huebner, R. J., Rowe, W. P., Schatten, W. E., Smith, R. R. \& Thomas, L. B. Studies on the use of viruses in the treatment of carcinoma of the cervix. Cancer 9, 1211-1218 (1956)
127. Laborda, E., Puig-Saus, C., Cascallo, M., Chillon, M. \& Alemany, R. Adeno-associated virus enhance wild-type and oncolytic adenovirus spread. Hum. Gene Ther. Methods 24, 372-380 (2013).

128. Kim, K. H. et al. A phase I clinical trial of Ad5/3- $\Delta 24$ a novel serotype-chimeric, infectivity-enhanced, conditionally-replicative adenovirus (CRAd), in patients with recurrent ovarian cancer. Gynecol Oncol. 130, 518-524 (2013).

129. Dmitriev, I. et al. An adenovirus vector with genetically modified fibers demonstrates expanded tropism via utilization of a coxsackievirus and adenovirus receptor-independent cell entry mechanism. J. Virol. 72, 9706-9713 (1998)

130. Shayakhmetov, D. M. \& Lieber, A. Dependence of adenovirus infectivity on length of the fiber shaft domain. J. Virol. 74, 10274-10286 (2000)

131. Takagi-Kimura, M. et al. Enhanced antitumor efficacy of fiber-modified, midkine promoter-regulated oncolytic adenovirus in human malignant mesothelioma. Cancer Sci. 104, 1433-1439 (2013).

132. Chen, G. X. et al. Clinical utility of recombinant adenoviral human p53 gene therapy: current perspectives. OncoTargets Ther. 7, 1901-1909 (2014).

133. Xia, Z J et al. Phase III randomized clinical trial of intratumoral injection of E1B gene-deleted adenovirus (H101) combined with cisplatin-based chemotherapy in treating squamous cell cancer of head and neck or esophagus. Ai Zheng 23, 1666-1670 (in Chinese) (2004).

134. Xu, R., Johnson, A. J., Liggitt, D. \& Bevan, M. J. Cellular and humoral immunity against vaccinia virus infection of mice. J. Immunol. 172, 6265-6271 (2004).

135. Tang, J. et al. Human T-cell responses to vaccinia virus envelope proteins. J. Virol. 80, 10010-10020 (2006)

136. Kim, J. H. et al. Systemic armed oncolytic and immunologic therapy for cancer with JX-594, a targeted poxvirus expressing GM-CSF. Mol. Ther 14, 361-370 (2006)

137. Scholl, S. M et al. Recombinant vaccinia virus encoding human MUC1 and IL2 as immunotherapy in patients with breast cancer. J. Immunother. 23 , 570-580 (2000)

138. Cole, D. J. et al. Phase I study of recombinant CEA vaccinia virus vaccine with post vaccination CEA peptide challenge. Hum. Gene Ther. 7, 1381-1394 (1996).

139. Kaufman, H. L. et al. Targeting the local tumor microenvironment with vaccinia virus expressing B7. for the treatment of melanoma. J. Clin. Invest. 115 1903-1912 (2005).

140. Kaufman, H. L. et al. Local delivery of vaccinia virus expressing multiple costimulatory molecules for the treatment of established tumors. Hum. Gene Ther. 17, 239-244 (2006)

141. Park, S. H. et al. Phase Ib trial of biweekly intravenous Pexa-Vec (JX-594), an oncolytic and immunotherapeutic vaccinia virus in colorectal cancer. Mol. Ther. http://dx.doi.org/10.1038/mt.2015.109 (2015)

142. Heo, J. et al. Randomized dose-finding clinical trial of oncolytic immunotherapeutic vaccinia JX-594 in liver cancer. Nat. Med. 19, 329-336 (2013).

143. Miyamoto, S. et al. Coxsackievirus B3 is an oncolytic virus with immunostimulatory properties that is active against lung adenocarcinoma. Cancer Res. 72, 2609-2621 (2012)

144. Andtbacka, R. H. l.et al. Final data from CALM A phase II study of Coxsackievirus A21 (CVA21) oncolytic virus immunotherapy in patients with advanced melanoma. J. Clin. Oncol. 33, S9030 (2015).

145. Elankumaran, S. et al. Type I interferon-sensitive recombinant newcastle disease virus for oncolytic virotherapy. J. Virol. 84, 3835-3844 (2010).

146. Ito, Y., Nagai, $Y$. \& Maeno, K. Interferon production in mouse spleen cells and mouse fibroblasts (L cells) stimulated by various strains of Newcastle disease virus. J. Gen. Virol. 62, 349-352 (1982).

147. Washburn, B. \& Schirrmacher, V. Human tumor cell infection by Newcastle Disease Virus leads to upregulation of HLA and cell adhesion molecules and to induction of interferons, chemokines and finally apoptosis. Int. J. Oncol. 21, 85-93 (2002).

148. Ertel, C., Millar, N. S., Emmerson, P. T. Schirrmacher, V. \& von Hoegen, P. Viral hemagglutinin augments peptide-specific cytotoxic $T$ cell responses. Eur. J. Immunol. 23, 2592-2596 (1993).

149. Bian, H., Wilden, H., Fournier, P., Peeters, B. \& Schirrmacher, V. In vivo efficacy of systemic tumor targeting of a viral RNA vector with oncolytic properties using a bispecific adapter protein. Int. J. Oncol. 29, 1359-1369 (2006). 
150. Janke, M. et al. Recombinant Newcastle disease virus (NDV) with inserted gene coding for GM-CSF as a new vector for cancer immunogene therapy. Gene Ther. 14, 1639-1649 (2007)

151. Apostolidis, L., Schirrmacher, V. \& Fournier, P. Host mediated anti-tumor effect of oncolytic Newcastle disease virus after locoregional application Int. J. Oncol. 31, 1009-1019 (2007).

152. Pecora, A. L. et al. Phase I trial of intravenous administration of PV701, an oncolytic virus, in patients with advanced solid cancers. J. Clin. Oncol. 20, 2251-2266 (2002).

153. Minuk, G. Y., Paul, R. W. \& Lee, P. W. The prevalence of antibodies to reovirus type 3 in adults with idiopathic cholestatic liver disease. J. Med. Virol. 16, 55-60 (1985).

154. Minuk, G. Y. et al. Reovirus type 3 infection in patients with primary biliary cirrhosis and primary sclerosing cholangitis. J. Hepatol. 5, 8-13 (1987).

155. Bluming, A. Z. \& Ziegler, J. L. Regression of Burkitt's lymphoma in association with measles infection. Lancet 2, 105-106 (1971).

156. Pasquinucci, G. Possible effect of measles on leukaemia. Lancet 1, 136 (1971)

157. Taqi, A. M., Abdurrahman, M. B., Yakubu, A. M. $\delta$ Fleming, A. F. Regression of Hodgkin's disease after measles. Lancet 1, 1112 (1981).

158. Merrill, M. K. et al. Poliovirus receptor CD155-targeted oncolysis of glioma. Neuro Oncol. 6, 208-217 (2004).

159. Gromeier, M., Lachmann, S., Rosenfeld, M. R. Gutin, P. H. \& Wimmer, E. Intergeneric poliovirus recombinants for the treatment of malignant glioma. Proc. Natl Acad. Sci. USA 97, 6803-6808 (2000).

160. Merrill, M. K., Dobrikova, E. Y. \& Gromeier, M Cell-type-specific repression of internal ribosome entry site activity by double-stranded RNA-binding protein 76. J. Virol. 80, 3147-3156 (2006).

161. Merrill, M. K. \& Gromeier, M. The double-stranded RNA binding protein 76:NF45 heterodimer inhibit translation initiation at the rhinovirus type 2 internal ribosome entry site. J. Virol. 80, 6936-6942 (2006)

162. Lu, Y. C. et al. Replicating retroviral vectors for oncolytic virotherapy of experimental hepatocellular carcinoma. Oncol. Rep. 28, 21-26 (2012).

163. Hales, R. K. et al. Assessing oncologic benefit in clinical trials of immunotherapy agents. Ann. Oncol. 21, 1944-1951 (2010)

164. Small, E. J. et al. A phase I trial of intravenous CG7870, a replication-selective, prostate-specific antigen-targeted oncolytic adenovirus, for the treatment of hormone-refractory, metastatic prostate cancer. Mol. Ther. 14, 107-117 (2006).
165. Laurie, S. A et al. A phase 1 clinical study of intravenous administration of PV701, an oncolytic virus, using two-step desensitization. Clin. Cancer Res. 12, 2555-2562 (2006).

166. Haddad, D. et al. Imaging characteristics, tissue distribution, and spread of a novel oncolytic vaccinia virus carrying the human sodium iodide symporter. PLOS ONE 7, e41647 (2012).

167. Hasegawa, K. et al. Dual therapy of ovarian cancer using measles viruses expressing carcinoembryonic antigen and sodium iodide symporter. Clin. Cancer Res. 12, 1868-1875 (2006)

168. Dingli, D. et al. Image-guided radiovirotherapy for multiple myeloma using a recombinant measles virus expressing the thyroidal sodium iodide symporter. Blood 103, 1641-1646 (2004).

169. Kummer, C. et al. Multitracer positron emission tomographic imaging of exogenous gene expression mediated by a universal herpes simplex virus 1 amplicon vector. Mol. Imag. 6, 181-192 (2007).

170. Tjuvajev, J. G. et al. Noninvasive imaging of herpes virus thymidine kinase gene transfer and expression: a potential method for monitoring clinical gene therapy. Cancer Res. 56, 4087-4095 (1996).

171. Grampp, G. \& Ramanan, S. Managing unexpected events in the manufacturing of biologic medicines. BioDrugs 27, 305-316 (2013).

172. Chen, D. Safety assurance for biologics manufactured in mammalian cell cultures: a multitiered strategy. Adv. Biochem. Eng. Biotechnol. 139, 167-183 (2014)

173. Martin-Moe, S. et al. A new roadmap for biopharmaceutical drug product development: Integrating development, validation, and quality by design. J. Pharm. Sci. 100, 3031-3043 (2011).

174. European Directorate for the Quality of Medicines. Tests for extraneous agents in viral vaccines for human use. European Pharmacopoeia 4th ed 415-418 (Council of Europe, 2002). Guidelines established for the production of viruses used in vaccine production that may provide the precedent for oncolytic virus production.

175. European Directorate for the Quality of Medicines Tests for extraneous agents in viral vaccines for human use. European Pharmacopoeia 4th ed 148-149 (Council of Europe, 2002). Guidelines established for the production of viruses used in vaccine production that may provide the precedent for oncolytic virus production.
176. Lolkema, M. P et al. A phase I study of the combination of intravenous reovirus type 3 Dearing and gemcitabine in patients with advanced cancer. Clin. Cancer Res. 17, 581-588 (2011).

177. Harrow, S et al. HSV1716 injection into the brain adjacent to tumour following surgical resection of high-grade glioma: safety data and long-term survival Gene Ther. 11, 1648-1658 (2004).

178. Herman, J. R. et al. In situ gene therapy for adenocarcinoma of the prostate: a phase I clinical trial. Hum. Gene Ther. 10, 1239-1249 (1999).

179. Teh, B. S. et al. Phase $\mathrm{I} / \mathrm{Il}$ trial evaluating combined radiotherapy and in situ gene therapy with or without hormonal therapy in the treatment of prostate cancer - a preliminary report. Int. J. Radiat. Oncol. Biol. Phys. 51, 605-613 (2001).

180. Herbst, R. S. et al. Predictive correlates of response to the anti-PD-L1 antibody MPDL3280A in cancer patients. Nature 515, 563-567 (2014).

181. Bellucci, R. et al. Interferon- $\gamma$-induced activation of JAK1 and JAK2 suppresses tumor cell susceptibility to NK cells through upregulation of PD-L1 expression. Oncoimmunology 4, e 1008824 (2015).

182. Terawaki, S. et al. IFN- $\alpha$ directly promotes programmed cell death- 1 transcription and limits the duration of T cell-mediated immunity. J. Immunol. 186, 2772-2779 (2011).

\section{Acknowledgements}

This work was supported, in part, by the US National Cancer Institute Grant UM1 CA186716-01.

Competing interests statement

The authors declare competing interests: see Web version for details.

FURTHER INFORMATION

Guidance for Industry: Design and Analysis of Shedding Studies for Virus or Bacteria-Based Gene Therapy and Oncolytic Products: http://www.fda.gov/downloads/ BiologicsBloodVaccines/GuidanceComplianceRegulatory Information/Guidances/CellularandGeneTherapy/ UCM404087.pdf

SUPPLEMENTARY INFORMATION

See online article: $\mathbf{1}$ (table)

ALL LINKS ARE ACTIVE IN THE ONLINE PDF 


\section{CORRIGENDUM}

Oncolytic viruses: a new class of immunotherapy drugs

Howard L. Kaufman, Frederick J. Kohlhapp \& Andrew Zloza

Nature Reviews Drug Discovery 14, 642-662 (2015)

In the original article, some descriptions of the properties of viruses in Tables 1 and 2 were inaccurate; the tables have been updated to reflect this. Also, on page 656, Newcastle Disease Virus (NDV) was accidentally described as a double-stranded RNA virus. NDV is a single-stranded RNA virus.

The authors would like to acknowledge R. C. Hoeben and B. G. van den Hoogen for alerting them to these errors. 